\title{
Subcritical hybrid inflation in a generalized superconformal model
}

\author{
Yoshihiro Gunji and Koji Ishiwata \\ Institute for Theoretical Physics, Kanazawa University, Kanazawa 920-1192, Japan
}

(Received 24 May 2021; accepted 22 September 2021; published 23 December 2021)

\begin{abstract}
We study a generalized superconformal model that gives rise to a subcritical regime of $D$-term hybrid inflation. Exhibiting the model both in a Jordan frame and in the Einstein frame, the effective potential of the subcritical regime is derived in the Einstein frame. It turns out that the inflaton-waterfall field dynamics leads to various types of inflaton potential. Consequently, the tensor-to-scalar ratio is found to range from $10^{-4}\left(10^{-3}\right)$ to 0.1 for getting $60(50) e$-folds before the end of inflation.
\end{abstract}

DOI: $10.1103 /$ PhysRevD.104.123545

\section{INTRODUCTION}

Observations of the cosmic microwave background $(\mathrm{CMB})$ radiation have provided important clues to solving the mysteries of the Universe. One of the prominent facts revealed by $\mathrm{CMB}$ observations is the indication of inflation at the early stages of the Universe. Inflation is a paradigm that not only solves the horizon and flatness problems but also gives the primordial curvature perturbation leading to the large-scale structure of the present Universe (if dark matter exists). Many inflation models have been proposed so far, and some of them have already been excluded by the CMB observations. The latest results by the Planck Collaboration on the scalar spectral index $n_{s}$, the tensorto-scalar ratio $r$, and the scalar amplitude $A_{s}$ are $[1,2]$

$$
\begin{gathered}
n_{s}=0.9649 \pm 0.0042 \quad(68 \% \text { C.L. }), \\
r<0.10 \quad(95 \% \text { C.L. }), \\
A_{s}=2.100 \pm 0.030 \times 10^{-9} \quad(68 \% \text { C.L. }) .
\end{gathered}
$$

Among the inflation models, the $R^{2}$ Starobinsky model $[3,4]$ is a traditional and representative model that has good agreement with the observations. It is also known that similar predictions for $n_{s}$ and $r$ are obtained in the $\alpha$ attractor model with small $\alpha$ [5].

Recently, $D$-term hybrid inflation in a supersymmetric model has been revisited, and new features of this model have been unveiled. It was found that the $\alpha$ attractor appears in the superconformal version of the model [6], while a chaotic regime was discovered in the subcritical regime,

Published by the American Physical Society under the terms of the Creative Commons Attribution 4.0 International license. Further distribution of this work must maintain attribution to the author(s) and the published article's title, journal citation, and DOI. Funded by SCOAP ${ }^{3}$. where the inflaton field value gets smaller than the criticalpoint value of the hybrid inflation $[7,8] .{ }^{1}$ On top of that, the superconformal version of the model has turned out to have the subcritical regime, and it has both the feature of the $\alpha$ attractor and natural inflation [12]. Such multiple characteristics in the subcritical regime of $D$-term hybrid inflation are controlled by (approximate) symmetries of the Kähler potential and the superpotential. This may relate to the geometry of the metric. The inflation model under conformal symmetry has recently been studied in metric-affine geometry instead of Riemannian geometry. It was shown that the $\alpha$ attractor and natural inflation emerge depending on the global symmetry imposed on the model [13]. Furthermore, $k$-inflation is studied in the conformal metricaffine geometry [14].

The subcritical hybrid inflation has other phenomenological features. It is free from the cosmic string problem due to the fact that the inflation continues long enough during the subcritical regime $[7,8]$. Besides, it can be embedded to the minimal supersymmetric standard model (and its extension) to give rich phenomenology, such as producing baryon asymmetry and dark matter and a characteristic pattern of neutrino masses [15]. Therefore, it is worth investigating the subcritical regime in the broad class of the inflation model.

In our paper, we study the subcritical regime of $D$-term hybrid inflation in a generalized superconformal model. While the model is formulated in the Einstein frame in the framework of the supergravity model, we show that it can be formulated in the extended form of the canonical superconformal supergravity model. Then, we discuss the dynamics of the inflaton and waterfall fields. It is shown that the subcritical regime is found in a wide range of parameter space, and that various forms of the effective

\footnotetext{
${ }^{1}$ References [9-11] also point out that inflation lasts below the critical point. In the model of the literature, the inflation is induced by a slow-rolling waterfall field.
} 
potential for inflation are derived. Consequently, the tensorto-scalar ratio turns out to be larger than $\mathcal{O}\left(10^{-4}\left(10^{-3}\right)\right)$ for 60 (50) $e$-folds before the end of inflation. In addition, it is found that the symmetry-enhanced points-namely, the Kähler potential without an explicit superconformal breaking term or integer value for $3 \alpha$ that relates to the dimension of the compactified space in superstring theory-are consistent with the Planck observation.

The results indicate further possibility to build a more phenomenologically viable model. One example is the minimal supersymmetric standard model (MSSM) augmented by the right-handed neutrinos, which was partly analyzed in Ref. [15]. In this model, one of the right-handed sneutrinos plays the role of an inflaton field, and the righthanded (s)neutrino generates the baryon number of the Universe. In the analysis of the reference, however, the neutrino oscillation data are not fully taken into account; meanwhile, the neutrino sector has been intensively studied by neutrino oscillation experiments [16-21] and cosmological observations $[1,22]$. Those data not only constrain such a model but also may give important hints for the new symmetry of the flavor. Non-Abelian discrete symmetry, such as $S_{3}, A_{4}, S_{4}$, and $A_{5}$, is one of the viable possibilities in that direction; it has been rigorously studied [23-29] to explain the mysterious pattern of the neutrino mixing and mass hierarchy. Thus, the results obtained in the present work would give a hint towards unveiling the underlying symmetry among inflation, baryogenesis, and the neutrino sector.

This paper is organized as follows: In Sec. II, the $D$-term hybrid inflation in the generalized superconformal model is formulated in a Jordan frame and in the Einstein frame. Dynamics of the inflaton and waterfall fields are discussed in Sec. III, and consequently the effective inflaton potential in the subcritical regime is derived. The cosmological consequences are discussed in Sec. IV. Section V contains our conclusions and discussion for future work. Throughout this paper, we use the Planck unit, $M_{\mathrm{pl}}=1$, unless otherwise stated, and the metric tensor $g_{\mu \nu}$ that gives $\eta_{\mu \nu}=\operatorname{diag}(-1,1,1,1)$ in the flat limit.

\section{THE MODEL}

We consider a generalized version of the canonical superconformal supergravity (CSS) model. The CSS model is proposed in Ref. [30]. The model is characterized by two components, the superconformal Kähler potential $\mathcal{N}$ and superconformal superpotential $\mathcal{W}$. In our paper, we introduce an additional parameter $\alpha(>0)$ in the superconformal Kähler potential:

$$
\begin{aligned}
\mathcal{N}= & -\left|X^{0}\right|^{2}\left[1-\frac{\left|S_{+}\right|^{2}+\left|S_{-}\right|^{2}+|N|^{2}}{\left|X^{0}\right|^{2}}\right. \\
& \left.-\frac{\chi}{2\left|X^{0}\right|^{2}}\left(\frac{N^{2} \overline{X^{0}}}{X^{0}}+\frac{\bar{N}^{2} X^{0}}{\bar{X}^{0}}\right)\right]^{\alpha},
\end{aligned}
$$

where we have introduced a real constant ${ }^{2} \chi$. A similar form of $\mathcal{N}$, but with $\chi=0$, is proposed in the context of the superconformal $\alpha$ attractor [5]. Here $X^{0}, S_{ \pm}$, and $N$ are chiral superfields that have the local U(1) charges $0, \pm q$ $(q>0)$, and 0 , respectively. In our paper, we use the same symbol for a chiral superfield and its scalar field unless otherwise noticed. ( $N$ and $S_{+}$will be identified as the inflaton and waterfall fields, respectively.) A nonzero $\chi$ explicitly breaks the superconformal symmetry. For the superconformal superpotential, on the other hand, we consider a renormalizable Yukawa interaction,

$$
\mathcal{W}=\lambda S_{+} S_{-} N,
$$

where $\lambda$ is a dimensionless constant. Here we ignore possible gauge-invariant and renormalizable terms, such as $S_{+} S_{-} X^{0}, N^{3}, X^{0} N^{2}$, etc., and stick to the simple model. ${ }^{3}$ After gauge fixing $X^{0}=\bar{X}^{0}=\sqrt{3}$ of the superconformal symmetry, the Lagrangian (in a Jordan frame) is obtained as

$\frac{\mathcal{L}_{J}}{\sqrt{-g_{J}}}=\mathcal{N}\left(-\frac{1}{6} R_{J}+\mathcal{A}_{\mu}^{2}\right)-\mathcal{N}_{\beta \bar{\beta}} g_{J}^{\mu \nu} \mathcal{D}_{\mu} z^{\beta} \mathcal{D}_{\nu} \bar{z}^{\bar{\beta}}-V_{J}$,

where $R_{J}$ is the Ricci scalar, $\mathcal{N}_{\beta \bar{\beta}} \equiv \partial^{2} \mathcal{N} / \partial z^{\beta} \partial \bar{z}^{\bar{\beta}}$ $\left(z^{\beta}=S_{ \pm}, N\right), g_{J \mu \nu}$ is the metric tensor, and $\mathcal{D}_{\mu} \equiv \partial_{\mu}-$ $i g Q A_{\mu}$ is the covariant derivative. Meanwhile, the auxiliary gauge field $\mathcal{A}_{\mu}$ defined in Refs. [30,31] has been introduced, and $\mathcal{A}_{\mu}$ can be taken to be zero when the scalar part is discussed as described in the literature. $A_{\mu}, g$, and $Q$ are the gauge field, the coupling, and the charge of the U(1) gauge. $V_{J}=V_{J}^{F}+V_{J}^{D}$ is the scalar potential in the Jordan frame, where

$$
\begin{gathered}
V_{J}^{F}=\mathcal{N}^{\beta \bar{\beta}} \mathcal{W}_{\beta} \overline{\mathcal{W}}_{\bar{\beta}}, \\
V_{J}^{D}=\frac{1}{2}(\operatorname{Re} f)^{a b} \mathcal{P}_{a} \mathcal{P}_{b} .
\end{gathered}
$$

Here, $\mathcal{N}^{\beta \bar{\beta}}$ is the $(\beta, \bar{\beta})$ component of the inverse of $\mathcal{N}_{I \bar{J}}$ $\left(X^{I}=X^{0}, S_{ \pm}, N\right)$, which is defined before gauge fixing; $\mathcal{W}_{\beta} \equiv \partial \mathcal{W} / \partial z^{\beta} ; f$ is the gauge kinetic function; and $\mathcal{P}_{a}=$ $-\eta_{a}^{\beta} \mathcal{N}_{\beta}-\tilde{\xi}\left(\mathcal{N}_{\beta} \equiv \partial \mathcal{N} / \partial z^{\beta}\right) . \eta_{a}^{\beta}$ is the Killing vector, and in the present case $\eta_{a}^{\beta}=Q g z^{\beta}$ and $f=1$. Since we consider $\mathrm{U}$ (1) theory, we omit the index $a$ hereafter. Note that we have introduced the Fayet-Iliopoulos (FI) term $\tilde{\xi}$ by adopting the procedure in Ref. [32]. According to the literature, an additional term in the Lagrangian is considered:

\footnotetext{
${ }^{2}$ In general, the term proportional to $\chi$ can be a more complicated form, as shown in Ref. [30].

${ }^{3}$ Such extension would be interesting from a phenomenological point of view. For example, $N$ can be identified as a righthanded neutrino that has Majorana mass in Ref. [15] when $\alpha=1$.
} 


$$
\frac{\Delta \mathcal{L}_{J}}{\sqrt{-g_{J}}}=g \frac{-\mathcal{N} \xi}{3} \mathcal{P}
$$

where $\xi$ is a constant. We take $\xi>0$ without the loss of generality. This term gives $\tilde{\xi}=g \mathcal{N} \xi / 3$ to get $\mathcal{P}=$ $-g Q z^{\beta} \mathcal{N}_{\beta}-g \mathcal{N} \xi / 3$.

The Lagrangian in the Einstein frame is obtained by the Weyl transformation,

$$
g_{J \mu \nu}=\left(-\frac{\mathcal{N}}{3}\right)^{-1} g_{E \mu \nu}
$$

where $g_{E \mu \nu}$ is the metric in the Einstein frame. Then we obtain

$$
\frac{\mathcal{L}_{E}}{\sqrt{-g_{E}}}=\frac{1}{2} R_{E}-K_{\beta \bar{\beta}} g_{E}^{\mu \nu} \mathcal{D}_{\mu} z^{\beta} \mathcal{D}_{\nu} \bar{z}^{\bar{\beta}}-V_{E}
$$

where $R_{E}$ is the Ricci scalar in the Einstein frame, and

$$
\begin{gathered}
K=-3 \alpha \ln \left(-\frac{\Phi}{3}\right), \\
\Phi=-3+\left|S_{+}\right|^{2}+\left|S_{-}\right|^{2}+|N|^{2}+\frac{\chi}{2}\left(N^{2}+\bar{N}^{2}\right), \\
V_{E}=\left(-\frac{\mathcal{N}}{3}\right)^{-2} V_{J},
\end{gathered}
$$

and $K_{\beta \bar{\beta}} \equiv \partial^{2} K / \partial z^{\beta} \partial \bar{z}^{\bar{\beta}}$.

Although we have derived it from a Jordan frame, one can derive the Lagrangian in the Einstein frame starting from the Kähler potential [Eq. (12)] and superpotential [Eq. (5)] in the supergravity model. Then the $F$ and $D$ terms are derived from them as

$$
\begin{aligned}
V_{E}^{F}= & e^{K}\left(K^{\beta \bar{\beta}} D_{\beta} \mathcal{W} D_{\bar{\beta}} \overline{\mathcal{W}}-3|\mathcal{W}|^{2}\right) \\
= & \left(-\frac{\Phi}{3}\right)^{1-3 \alpha} \frac{1}{\alpha}\left[\delta^{\beta \bar{\beta}} \mathcal{W}_{\beta} \overline{\mathcal{W}}_{\bar{\beta}}+\frac{1}{\Delta}\left|\delta^{\beta \bar{\beta}} \mathcal{W}_{\beta} \Phi_{\bar{\beta}}-3 \alpha \mathcal{W}\right|^{2}\right. \\
& \left.+\frac{9 \alpha}{\Phi}(1-\alpha)|\mathcal{W}|^{2}\right] \\
& V_{E}^{D}=\frac{1}{2} D^{2}=\frac{g^{2}}{2}\left(K_{\beta} Q z^{\beta}-\xi\right)^{2} \\
& =\frac{g^{2}}{2}\left[\left(-\frac{\Phi}{3}\right)^{-1} \alpha q\left(\left|S_{+}\right|^{2}-\left|S_{-}\right|^{2}\right)-\xi\right]^{2}
\end{aligned}
$$

Here, $K^{\beta \bar{\beta}}$ is the inverse of $K_{\beta \bar{\beta}}, D_{\beta} \mathcal{W} \equiv \mathcal{W}_{\beta}+K_{\beta} \mathcal{W}$, $\mathcal{W}_{\beta} \equiv \partial \mathcal{W} / \partial z^{\beta}, \Phi_{\beta} \equiv \partial \Phi / \partial z^{\beta}$, and $\Delta \equiv \Phi-\delta^{\beta \bar{\beta}} \Phi_{\beta} \Phi_{\bar{\beta}}$. We have explicitly checked that the sum of Eqs. (15) and (16) coincides with Eq. (14). We note that when $\alpha=1$, this model reduces to the one studied in Refs. [6,32,33], as expected.
From the scalar potential, the masses of the scalar part of the canonically normalized $S_{ \pm}$are given by

$$
m_{ \pm}^{2}=\left(-\frac{\Phi}{3}\right)^{2-3 \alpha} \frac{\lambda^{2}}{\alpha^{2}}|N|^{2} \mp q g^{2} \xi
$$

As with canonical $D$-term hybrid inflation, $S_{+}$acquires a tachyonic instability depending on the value of $N$, while $S_{-}$ is stabilized at the origin. Hereafter, we take $S_{-}=0$. The critical-point value of $N$ where $S_{+}$becomes tachyonic is determined by $m_{+}=0$. Since both the real and imaginary parts of $N$ can play the role of inflaton in the model, we take $\phi \equiv \sqrt{2} \operatorname{Re} N$ as the inflaton field without the loss of generality. ${ }^{4}$ In addition, since the scalar potential depends on $\left|S_{+}\right|$, we define a field $s \equiv \sqrt{2}\left|S_{+}\right|$that we refer to as the waterfall field. Then, defining $\Phi(\phi, s)$ as

$$
\begin{aligned}
\Phi(\phi, s) & \left.\equiv \Phi\right|_{\sqrt{2} N=\sqrt{2} \bar{N}=\phi, \sqrt{2}\left|S_{+}\right|=s, S_{-}=0} \\
& =-3+\frac{1}{2}\left(s^{2}+(1+\chi) \phi^{2}\right)
\end{aligned}
$$

the critical-point value $\phi_{c}$ should satisfy

$$
\left(-\frac{\Phi_{c}}{3}\right)^{2-3 \alpha} \phi_{c}^{2}=\frac{2 \alpha^{2}}{k}
$$

where $\Phi_{c} \equiv \Phi\left(\phi_{c}, 0\right)$ and

$$
k \equiv \lambda^{2} / q g^{2} \xi
$$

The number of solutions of Eq. (19) depends on the values of $\alpha$ and $\chi$. If it has multiple solutions, the potential gets complicated, and it becomes different from the potential in the canonical hybrid inflation. In our study, we focus on the case where there is one critical point. In that case, the valid parameter space is ${ }^{5}$

$$
\left\{\begin{array}{l}
\text { (i) } \quad \chi<-1 \text { and }(0<) \alpha \leq 1 \\
\text { (ii) } \quad \chi>-1 \text { and } \alpha \geq 2 / 3
\end{array}\right.
$$

In the parameter space, $\alpha=1$ and $2 / 3$ are special values, since the critical-point values can be obtained analytically as

\footnotetext{
${ }^{4}$ To be explicit, the results for the case where $\operatorname{Im} N$ is the inflaton field are obtained by replacing $\chi$ with $-\chi$. In contrast to the previous studies $[8,33]$, we do not restrict our present study to the case of $\chi \simeq-1(+1)$ where $\operatorname{Re} N(\operatorname{Im} N)$ has an approximate shift symmetry. Here, "approximate" means that the shift symmetry in the Kähler potential is broken by the superpotential. Therefore, the mass of $N$ appears in general [see Eq. (24)].

${ }^{5}$ When $\chi=-1, \phi_{c}^{2}=2 \alpha^{2} / k$ for any value of $\alpha$. This case corresponds to the subcritical hybrid inflation with shift symmetry, which is already studied in Refs. $[7,8]$.
} 


$$
\phi_{c}=\left\{\begin{array}{ll}
\sqrt{6 /(3 k+1+\chi)} & \text { for } \alpha=1 \\
(2 / 3) \sqrt{2 / k} & \text { for } \alpha=2 / 3
\end{array}\right. \text {. }
$$

It is obvious that $k$ has a bound:

$$
k>\left\{\begin{array}{ll}
-(1+\chi) / 3 & \text { for } \alpha=1 \text { and } \chi<-1 \\
4(1+\chi) / 27 & \text { for } \alpha=2 / 3 \text { and } \chi>-1
\end{array} .\right.
$$

The latter is given by $\phi_{c}<\phi_{\max } \equiv \sqrt{6 /(1+\chi)}$, where $\phi_{\max }$ is determined by $\Phi\left(\phi_{\max }, 0\right)=0$.

For successful inflation, the stabilization of the imaginary part of $N$, which we define as $\tau \equiv \sqrt{2} \operatorname{Im} N$, should be guaranteed in the subcritical region where $\phi<\phi_{c}$. The mass of $\tau$ in the region is given by

$$
\begin{aligned}
m_{\tau}^{2}= & \frac{g^{2} \xi^{2} k}{\alpha^{2}}\left(-\frac{\Phi_{0}}{3}\right)^{1-3 \alpha}(1-\Psi(\phi)) \\
& \times\left[1-\frac{\phi^{2}}{6}\{3-\chi+3 \alpha(\chi-1)\}\right],
\end{aligned}
$$

where $\Phi_{0} \equiv \Phi(\phi, 0)$ and

$$
\Psi(\phi) \equiv\left(\frac{\Phi_{0}}{\Phi_{c}}\right)^{2-3 \alpha} \frac{\phi^{2}}{\phi_{c}^{2}}=\frac{k}{2 \alpha^{2}}\left(-\frac{\Phi_{0}}{3}\right)^{2-3 \alpha} \phi^{2}
$$

which satisfies $\Psi\left(\phi_{c}\right)=1$. Here $s^{2} \ll \phi^{2}$ has been used, which will be validated in the later discussion. To satisfy $m_{\tau}^{2}>0$ in the valid parameter space above, we find the following preferred regions:

(i) $\chi<-1$ :

$$
\left\{\begin{array}{c}
1 / 3+2 / 3(1-\chi)<\alpha \leq 1 \\
\alpha<1 / 3+2 / 3(1-\chi) \\
\text { depending on other parameters }
\end{array} .\right.
$$

(ii) $\chi>-1$ :

$$
\left\{\begin{array}{l}
\chi<1 \\
\chi \gg 1 \text { and small } \alpha(\text { but } \geq 2 / 3)
\end{array} .\right.
$$

In our numerical analysis, we will compute the cosmological consequences in the parameter space given in Eqs. (21) and (22) and see consistency with the above regions. We will see that $m_{\tau}^{2}>0$ gives a constraint for the $\chi \gtrsim 5$ case. When $\tau$ is stabilized to the origin, the scalar potential after the critical point is given by

$$
\begin{aligned}
V_{\mathrm{tot}}(\phi, s)= & V_{E}^{F}+V_{E}^{D} \\
= & \left(-\frac{\Phi(\phi, s)}{3}\right)^{1-3 \alpha} \frac{\lambda^{2}}{4 \alpha} \phi^{2} s^{2} \\
& +\frac{g^{2}}{8}\left[\left(-\frac{\Phi(\phi, s)}{3}\right)^{-1} \alpha q s^{2}-2 \xi\right]^{2} .
\end{aligned}
$$

Even if $\tau$ is stabilized at the origin, it has a quantum fluctuation during inflation, which might cause another instability. In the model, $-\Phi$ must be positive. Therefore, this requirement leads to a constraint on the amplitude of $\tau$ :

$$
\tau^{2}<\frac{6}{1-\chi}-\frac{1+\chi}{1-\chi} \phi^{2}
$$

for $\chi<1$. (There is no constraint when $\chi \geq 1$.) The quantum fluctuation during inflation is estimated as

$$
\tau^{2} \sim \frac{H^{4}}{m_{\tau}^{2}} \sim g^{2} \xi^{2}
$$

where $H$ is the Hubble parameter. Here we have used Eq. (24) and $H \sim g^{2} \xi^{2}$. Though $m_{\tau}=0$ at the critical point, there is a mass term that arises at loop level (see the next section). Even in that case, the estimate for the mass is roughly the same except for an extra loop factor. In the allowed parameter space that we will show in the later analysis, this quantity is extremely smaller than unity. Therefore, the condition for Eq. (29) is always satisfied. One may worry about the isocurvature induced by $\tau$. We note that since $\tau$ has the same order of decay width as $\phi$ has, $\tau$ decays at the time of reheating. Assuming that $\tau$ dominantly decays to a radiation bath, the isocurvature that $\tau$ produces has no effect on the later thermal history. In addition, the energy density of $\tau$ during inflation is estimated as $m_{\tau}^{2} \tau^{2} \sim H^{4}$, which is much smaller than the inflaton energy density. Then, inflation driven by the $\phi-s$ system is not affected by the quantum fluctuation of $\tau$. Thus, there is no constraint due to the quantum fluctuation ${ }^{6}$ of $\tau$.

\section{DYNAMICS OF INFLATON AND WATERFALL FIELDS}

We discuss the dynamics of inflaton and waterfall fields around and after the critical point. In the typical hybrid inflation model, inflation ends at the critical point where the waterfall field becomes tachyonic. In the subcritical hybrid inflation, by contrast, inflation continues after the critical point. The crucial point here is a suppressed $\lambda$, as pointed out in Ref. [7]. If $\lambda \ll 1$,

\footnotetext{
${ }^{6} \mathrm{We}$ are indebted to Tomo Takahashi for private communication on this issue.
} 
therefore, the subcritical regime is expected to emerge in the generalized framework of the superconformal hybrid inflation model. In this section, we confirm this and give the effective potential for inflation after the critical point. See Refs. [8,32,33] for details.

Before it reaches the critical point, we assume that the inflaton field slowly rolls down to the critical point while the other scalar fields are initially stabilized at the origin. Since the tree-level potential is constant, the motion of the inflaton field is driven by the Coleman-Weinberg potential $[7,34]$, which is given by

$$
V_{1 L}=\frac{g^{4} q^{2} \xi^{2}}{32 \pi^{2}} L(\Psi),
$$

where $\quad L(x) \equiv(x-1)^{2} \ln (x-1)+(x+1)^{2} \ln (x+1)-$ $2 x^{2} \ln x-\ln 16$. The velocity of the inflaton field at the critical point $\dot{\phi}_{c}$ is given by

$$
\dot{\phi}_{c}=-\left.\frac{1}{3 H_{c}} \frac{\partial V_{1 L}}{\partial \phi}\right|_{\phi=\phi_{c}},
$$

where $H_{c}$ is the Hubble parameter at the critical point and the dot signifies a time derivative. After the critical point, the tachyonic growth of the waterfall field begins. In order to describe the dynamics, we use the canonically normalized waterfall field. Taking $\hat{s}_{k}$ as a Fourier mode of the canonically normalized waterfall field, the equation of motion is given by $[32,33,35]^{7}$

$$
\ddot{\hat{s}}_{k}+\left(k^{2} e^{-2 H_{c} t}-\frac{9}{4} H_{c}^{2}-\hat{d}^{3} t\right) \hat{s}_{k}=0,
$$

where $\hat{d}$ is obtained as

$$
\hat{d}^{3}=\frac{g^{2} q \xi\left(2-(1-\alpha)(1+\chi) \phi_{c}^{2}\right)}{\phi_{c}\left(-\Phi_{c} / 3\right)}\left|\dot{\phi}_{c}\right| .
$$

It is noted that $\hat{d}>0$ and $-\dot{\phi}_{c}>0$ are satisfied in the parameter space of Eqs. (21) and (22). By solving the equation of motion, we get the variance $\left\langle\hat{s}^{2}(t)\right\rangle$. After the decoherence time $t_{\mathrm{dec}}$ defined in Ref. [7], the variance is matched to the classical field, and the time evolutions of the waterfall field and inflaton field are determined by the classical equations of motion. Namely, we solve the classical equations of motion of $\phi$ and $s$ with a boundary condition $s\left(t_{\mathrm{dec}}\right)=\sqrt{\left\langle\hat{s}^{2}\left(t_{\mathrm{dec}}\right)\right\rangle / K_{S_{+} \bar{S}_{+}, c}}$ :

$$
\dot{\phi}+\frac{1}{3 H K_{N \bar{N}}} \frac{\partial V_{\text {tot }}}{\partial \phi}=0,
$$

\footnotetext{
${ }^{7}$ Here, $k$ stands for momentum. Do not confuse this with the dimensionless parameter $k$ given in Eq. (20).
}

$$
\dot{s}+\frac{1}{3 H K_{S_{+} \bar{S}_{+}}} \frac{\partial V_{\mathrm{tot}}}{\partial s}=0,
$$

where

$$
\begin{gathered}
H=\sqrt{V_{\mathrm{tot}}(\phi, s) / 3} \\
K_{N \bar{N}}=\alpha \frac{1+\frac{1}{6} \chi(1+\chi) \phi^{2}-\frac{1}{6} s^{2}}{(-\Phi(\phi, s) / 3)^{2}}, \\
K_{S_{+} \bar{S}_{+}}=\alpha \frac{1-\frac{1}{6}(1+\chi) \phi^{2}}{(-\Phi(\phi, s) / 3)^{2}} .
\end{gathered}
$$

Here $K_{S_{+} \bar{S}_{+}, c}$ is the value at the critical point.

In order to track the inflation dynamics in the $\phi$-s system, we define the slow-roll parameters by the Hubble parameter,

$$
\epsilon_{H} \equiv-\frac{\dot{H}}{H^{2}}, \quad \eta_{H} \equiv \epsilon_{H}-\frac{\ddot{H}}{2 H \dot{H}} .
$$

We have confirmed that inflation continues after crossing the critical point in the parameter space we are interested in. In addition, it is found that the dynamics after the critical point is effectively described by the single field. To be explicit, the waterfall field is relaxed to the local minimum $s_{\min }$ after up to a few Hubble times. During this period, the inflaton field merely moves and stays at the critical point. The local minimum value is approximately given by ${ }^{8}$

$$
s_{\min }^{2}(\phi)=-\frac{\Phi_{0}}{3} \frac{2 \xi}{q \alpha}(1-\Psi(\phi)) .
$$

We have numerically checked that mass of $s$ is larger than the Hubble in the subcritical region. Namely, the same situation in Refs. [7,8,33] is realized. Putting $s_{\min }$ into $V_{\text {tot }}(\phi, s)$ and ignoring parametrically unimportant terms suppressed by $\xi$, the effective potential in the subcritical regime is obtained as

$$
V(\phi)=g^{2} \xi^{2} \Psi(\phi)\left(1-\frac{1}{2} \Psi(\phi)\right) .
$$

We have confirmed that after the waterfall field relaxes to the local minimum, $\epsilon_{H}$ and $\eta_{H}$ coincide with $\epsilon(\phi)$ and $\eta(\phi)$ defined in Eq. (44), respectively, derived from the single-field effective potential $V(\phi)$. In later analysis, we

\footnotetext{
${ }^{8}$ To be precise, the local minimum should be determined numerically by solving $\partial V_{\text {tot }}(\phi, s) / \partial s=0$. However, it turns out that Eq. (41) agrees well with the exact solution when $\xi \ll 1$, which is the parameter region on which we focus.
} 

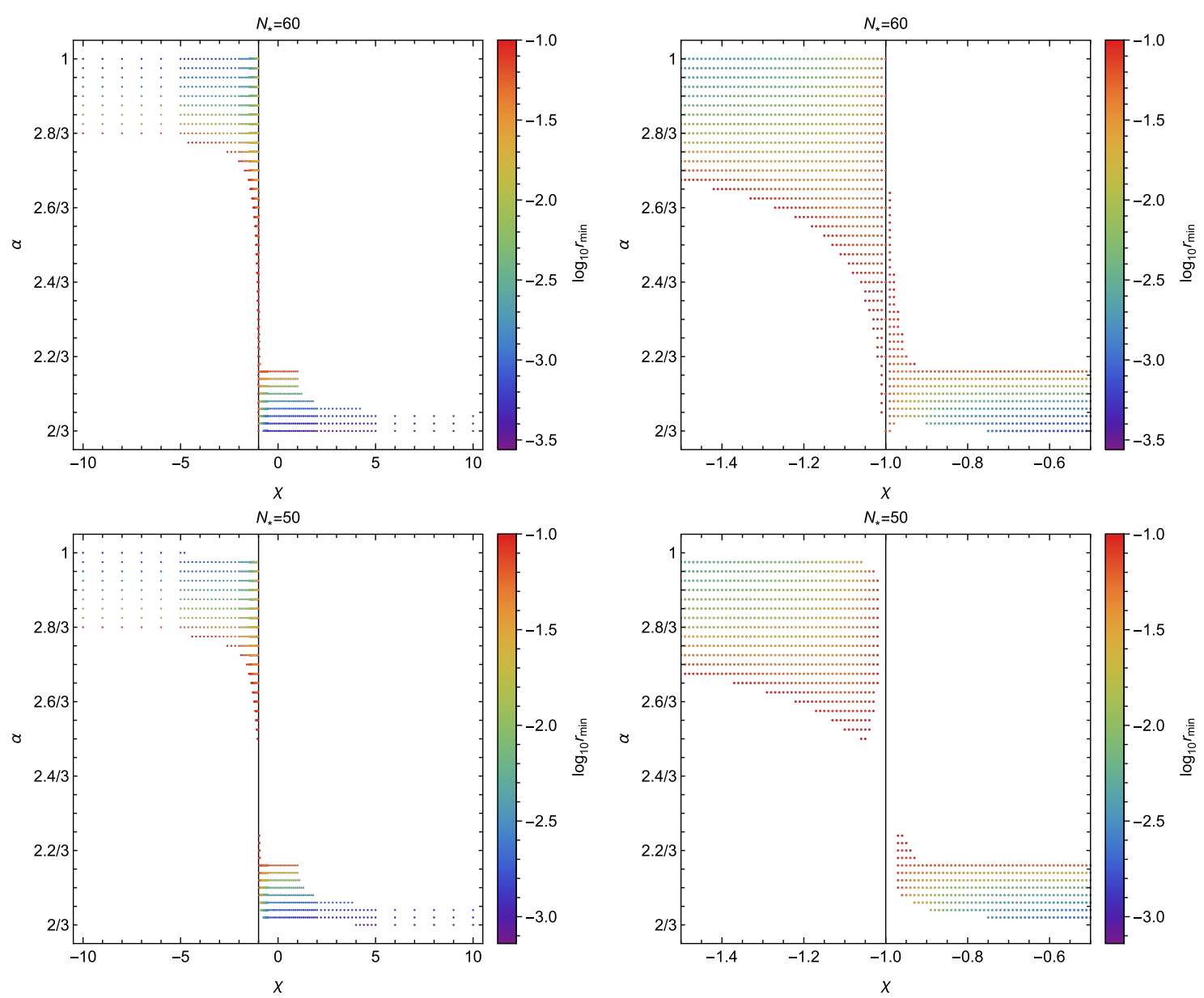

FIG. 1. Allowed region for $\alpha$ and $\chi$ for $N_{*}=60$ (top) and 50 (bottom) by imposing $m_{\tau}^{2}>0$ and Eqs. (1)-(3). The right panels are the same as the left ones, but the range of $-1.5 \leq \chi \leq-0.5$ is magnified. The color map indicates the minimum value of the predicted tensor-to-scalar ratio, $r_{\min }$.

use the effective potential to discuss the cosmological consequences. We will see in the next section that the typical inflaton field value that is canonically normalized is super-Planckian. However, the predicted tensor-toscalar ratio can be much smaller than unity.

Careful readers may worry about the effect of the waterfall field on the adiabatic curvature perturbation. It is expected to be negligible since, as we will see in the next section, the trajectory of the subcritical inflation is almost straight along the inflaton field. Such trajectory was already studied and analyzed in Ref. [7]. ${ }^{9}$ The effect can be evaluated by calculating a quantity $e^{\beta}=1+4 \eta_{\perp}^{2} H^{2} / M^{2}$ given in Ref. [36] (see also Ref. [37].) Here we follow the

\footnotetext{
${ }^{9}$ The inflation trajectory below the critical point is also analyzed in Refs. [9-11]. In that case, the trajectory during inflation is almost the waterfall field direction.
}

notation of Ref. [36]. (Do not confuse their $\beta$ with the one defined in this paper.) A variable $\eta_{\perp}$ describes the curvature of the trajectory. Namely, $\eta_{\perp}=0$ means that the trajectory is straight. $M$ is the mass of the field perpendicular to the inflaton direction. The value $e^{\beta}$ has an impact on the scalar amplitude as

$$
A_{s} \rightarrow A_{s}^{\prime}=e^{\beta} A_{s}
$$

Using the formula given in Ref. [36], we have found that $\eta_{\perp}^{2} \sim \mathcal{O}\left(10^{-6}\right)$ around $60 e$-folds in the valid parameter regions that will be shown in Fig. 1. In addition, $H^{2} / M^{2} \sim \xi$, which is suppressed as $\mathcal{O}\left(10^{-4}\right)$ (see Figs. 2, 5, and 8). Consequently, $e^{\beta}-1 \sim \mathcal{O}\left(10^{-10}\right)$, which is sufficiently small. Therefore, the effective description in the subcritical regime is valid. 

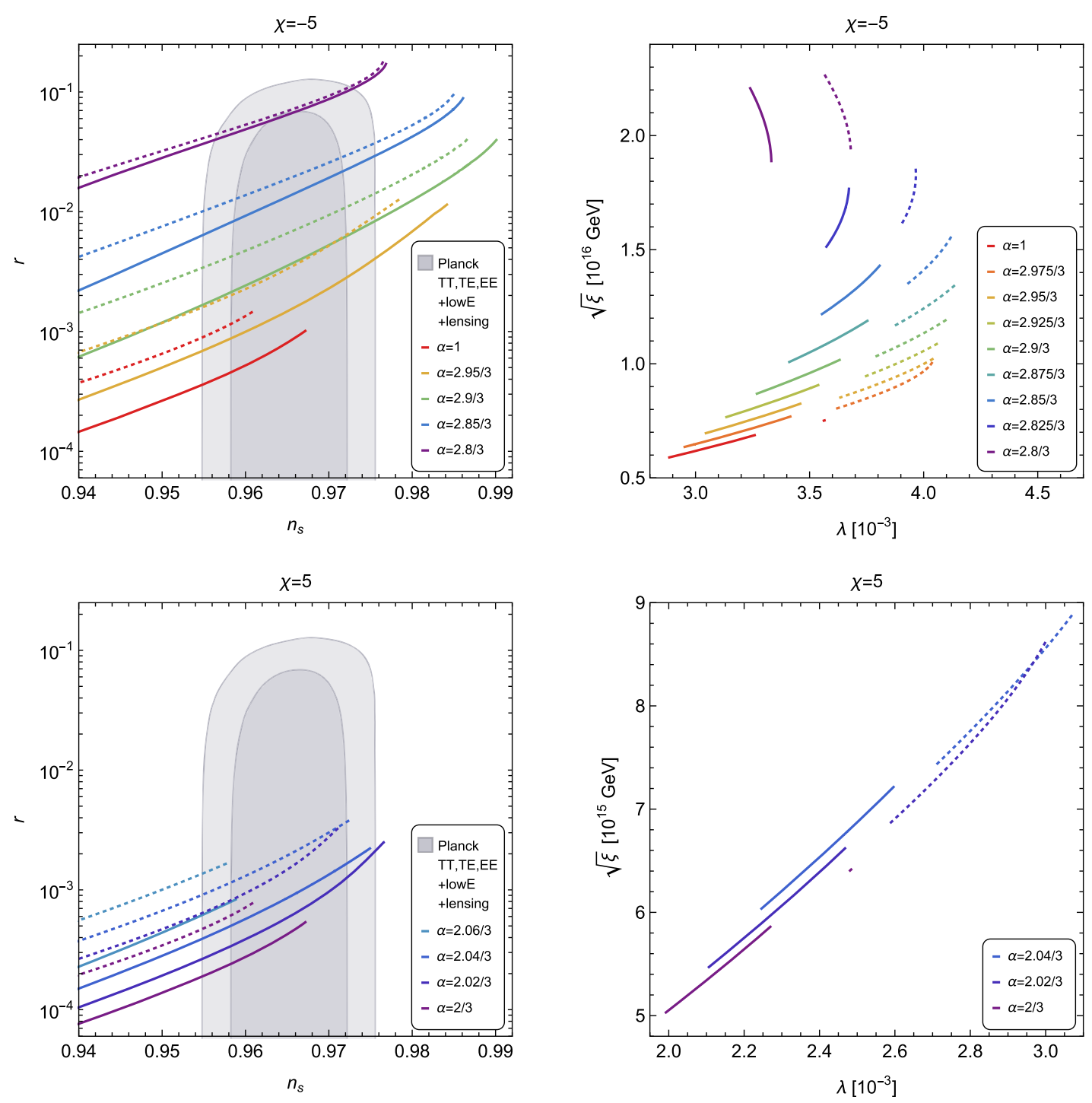

FIG. 2. Left: Predicted scalar spectral index and tensor-to-scalar ratio for fixed $e$-folds, $N_{*}=60$ (solid curves) and 50 (dotted curves), and various values of $\alpha$. In the top and bottom panels, $\chi$ is taken to -5 and 5, respectively. We impose $m_{\tau}^{2}>0$, and $1 \sigma$ (dark shaded) and $2 \sigma$ (light shaded) regions from the Planck Collaboration [2] are also shown. Right: Allowed regions for $\lambda$ and $\sqrt{\xi}$ for $\chi=-5$ (top) and 5 (bottom), by imposing Eqs. (1)-(3).

\section{COSMOLOGICAL CONSEQUENCES}

Now, we are ready to discuss cosmological consequences of this model. We compute the scalar spectral index, the tensor-to-scalar ratio, and the scalar amplitude and compare them with the latest observational results [Eqs. (1)-(3)].

\section{A. Cosmological parameters and overview of the results}

The slow-roll parameters defined by the effective potential are

$$
\epsilon(\phi) \equiv \frac{1}{2}\left(\frac{V^{\prime}}{V}\right)^{2}, \quad \eta(\phi) \equiv \frac{V^{\prime \prime}}{V}
$$

Here, $V^{\prime}=d V / d \hat{\phi}$ and $V^{\prime \prime}=d^{2} V / d \hat{\phi}^{2}$, and $\hat{\phi}$ is the canonically normalized inflaton field, which is defined by

$$
\frac{d \phi}{d \hat{\phi}}=\left.K_{N \bar{N}}^{-1 / 2}\right|_{s=s_{\min }}
$$

Since the field value of the waterfall field is found to be parametrically much smaller than the inflaton field value, it can be approximated as $s_{\min } \simeq 0$ during inflation. Therefore,

$$
K_{N \bar{N}} \simeq \frac{3 \alpha}{-\Phi_{0}}\left[1+\frac{(1+\chi)^{2} \phi^{2}}{-2 \Phi_{0}}\right]
$$

Inflation ends at 


$$
\phi=\phi_{\text {end }} \equiv \operatorname{Max}\left\{\phi_{\epsilon}, \phi_{\eta}\right\} .
$$

$\phi_{\epsilon}$ and $\phi_{\eta}$ are determined by $\epsilon\left(\phi_{\epsilon}\right)=1$ and $\left|\eta\left(\phi_{\eta}\right)\right|=1$, respectively. $n_{s}, r$, and $A_{s}$ are determined by the slow-roll parameters as

$$
\begin{gathered}
n_{s}=1+2 \eta\left(\phi_{*}\right)-6 \epsilon\left(\phi_{*}\right), \\
r=16 \epsilon\left(\phi_{*}\right), \\
A_{s}=\frac{V\left(\phi_{*}\right)}{24 \pi^{2} \epsilon\left(\phi_{*}\right)} .
\end{gathered}
$$

Here, $\phi_{*}$ is determined by the number of $e$-folds before the end of inflation:

$$
N_{*}=\int_{\hat{\phi}_{\mathrm{end}}}^{\hat{\phi}_{*}} d \hat{\phi} \frac{V}{V^{\prime}}=\int_{\phi_{\mathrm{end}}}^{\phi_{*}} d \phi \sqrt{\frac{K_{N \bar{N}}}{2 \epsilon(\phi)}},
$$

where $\hat{\phi}_{*}$ and $\hat{\phi}_{\text {end }}$ are canonically normalized field values corresponding to $\phi_{*}$ and $\phi_{\text {end }}$, respectively. In our numerical analysis, we take $q=g=1$ without the loss of generality. This is due to the fact that when $\xi \ll 1$, which is the case we are interested in, $q$ and $g$ can be absorbed into $\lambda$ and $\xi$ by redefining these as $\bar{\lambda} \equiv \lambda / \sqrt{q g}$ and $\bar{\xi} \equiv g \xi$, respectively. This is easily seen in Eq. (42). Since $\xi$ is cancelled in the slow-roll parameters, $\phi_{\text {end }}$ and $\phi_{*}$ are determined for $\alpha, \chi, k$, and $N_{*}$, and they give $n_{s}$ and $r$. The median value of the observed scalar amplitude determines the value of $\lambda$ and $\xi$ (also using the value of $k$ ).

First of all, we give the allowed region on the $(\alpha, \chi)$ plane in Fig. 1. We impose $m_{\tau}^{2}>0$ and the observed results given in Eqs. (1)-(3). In the plot $N_{*}=60$ and 50 are taken, and each dot corresponds to an allowed point. For a given set of $\alpha, \chi$, and $N_{*}$, the values of $n_{s}$ and $r$ are given as a function of $k$. When $k$ has solutions such that the predicted $n_{s}$ and $r$ are within the range of Eqs. (1) and (2), a dot is plotted on the $\alpha-\chi$ plane. The color map shows the minimum value of $r$ in the range of Eqs. (1) and (2). It is found that selective regions are allowed, and the behavior changes around $\chi=-1$. In addition, the allowed value of $\alpha$ saturates for $|\chi| \gtrsim 5$. When $N_{*}=60$, for instance, the allowed regions are $\alpha \simeq 1$ for $\chi \lesssim-5,2 / 3 \leq \alpha \leq 1$ for $\chi \simeq-1$, and $\alpha \simeq 2 / 3$ for $\chi \gtrsim 5$, and the predicted $r$ changes by orders of magnitude. It is found that Eqs. (26) and (28) do not give constraints on the parameters for $\chi<-1$. On the contrary, the obtained allowed region roughly tracks the region indicated in Eqs. (27) and (30) for $\chi>-1$. To investigate further, we categorize the parameter space into two regions:

$$
\left\{\begin{array}{l}
|\chi| \gtrsim 5 \\
\chi \simeq-1
\end{array}\right.
$$

We examine the dynamics of the inflaton and waterfall fields and their consequences in detail in Secs. IV B and IV C. In addition, we further investigate the specific cases $\alpha=1,2 / 3$, and $\chi=0$ in Sec. IV D. These cases are motivated by theoretical models beyond supergravity. In superstring theory, $3 \alpha$ corresponds to the dimension of the compactified space. Therefore, it is supposed to be an integer. When $\chi=0$, on the other hand, the superconformal invariance is exact in the Kähler potential, and it corresponds to a symmetry enhanced point.

\section{B. $|\chi| \gtrsim 5$}

Summary of the predictions. - The predictions for $n_{s}$ and $r$ are shown in the left panels of Fig. 2 for $\chi=-5$ (top) and 5 (bottom) with various values of $\alpha$. Here the stability condition for $\tau$ is imposed. In the right panels, the parameters $(\sqrt{\xi}, \lambda)$ that are consistent with the Planck observations are plotted for $N_{*}=60$ and 50. For a given $\alpha$, it is found that $n_{s}$ and $r$ get larger for smaller $k$. For $\chi=-5$, $r$ tends to be smaller when $\alpha \rightarrow 1$; meanwhile, it can be as large as $\mathcal{O}(0.1)$. This is the opposite behavior compared to the $\alpha$ attractor model [5]. As a consequence, a lower bound is obtained as $r \gtrsim 10^{-3}$. For $\chi=5$, on the other hand, $r$ is found to be suppressed as $10^{-4} \lesssim r \lesssim 10^{-3}$, and it gets smaller as $\alpha$ decreases. The parameter space that is consistent with the Planck data turns out to be $10^{-3} \lesssim \lambda \lesssim$ $10^{-2}$ and $\sqrt{\xi} \sim \mathcal{O}\left(10^{16} \mathrm{GeV}\right)$. The results for $\chi<-5$ $(\chi>5)$ behave almost the same as those ${ }^{10}$ for $\chi=$ $-5(\chi=5)$.

To get a better understanding of the results, it is legitimate to describe the effective potential in terms of the canonically normalized field $\hat{\phi}$. In addition, we consider a large field value limit for $\phi$ to give analytical expressions for the effective potential. Even though the analytical expressions are not always valid, they are used to help understand the numerical results for $n_{s}$ and $r$ qualitatively.

When $|\chi| \gg 1$ and in a large field limit, the second term in the parenthesis in Eq. (46) can be neglected. In that case, the Kähler metric is approximately given by

$$
K_{N \bar{N}} \simeq \frac{3 \alpha(1+\chi)^{2} \phi^{2}}{2 \Phi_{0}^{2}}
$$

and consequently Eq. (45) can be solved analytically as to give $\phi$. Using the result, $\Phi_{0}$ and $\Psi$ are determined. The results are

$$
\begin{gathered}
\phi^{2} \simeq\left\{\begin{array}{ll}
\frac{1}{\beta}\left(C e^{\sqrt{\frac{2}{3 \alpha}} \hat{\phi}}-1\right) & (\chi<-1) \\
\frac{1}{-\beta}\left(1-C e^{-\sqrt{\frac{2}{3 \alpha}} \hat{\phi}}\right) & (\chi>-1)
\end{array},\right. \\
-\frac{\Phi_{0}}{3} \simeq \begin{cases}C e^{\sqrt{\frac{2}{3 \alpha}} \hat{\phi}} & (\chi<-1) \\
C e^{-\sqrt{\frac{2}{3 \alpha}} \hat{\phi}} & (\chi>-1)\end{cases}
\end{gathered}
$$

\footnotetext{
${ }^{10} \mathrm{We}$ find that the preferred value of $\lambda$ becomes larger, but it is less than $10^{-2}$.
} 
TABLE I. Approximated expression of $\Psi$ in the limit $\left|(1+\chi)^{2} \phi^{2} /\left(-2 \Phi_{0}\right)\right| \gg 1$. For cases of $\chi<-1 \& \alpha \neq 1$ and $\chi>-1 \& \alpha \neq 2 / 3,2 \alpha^{2}|\beta| / k \gg 1$ is further assumed. The parameter space where the approximated expression is valid is given in the right column. (See also Fig. 1 for the allowed region for $\alpha$ and $\chi$.) The effective potential can be obtained from $\Psi$ by using Eq. (42).

\begin{tabular}{lcc}
\hline \hline Cases & Approximated expression for $\Psi$ & Valid parameters \\
\hline$\chi<-1 \& \alpha \neq 1$ & $e^{\sqrt{\frac{6}{\alpha}}(1-\alpha)\left(\hat{\phi}-\hat{\phi}_{c}\right)}$ & $\chi \lesssim-20 \& \alpha \lesssim 2.8 / 3$ \\
$\chi>-1 \& \alpha \neq 2 / 3$ & $e^{\sqrt{\frac{2}{3 \alpha}}(3 \alpha-2)\left(\hat{\phi}-\hat{\phi}_{c}\right)}$ & $\chi \gtrsim-0.5 \& \alpha \gtrsim 2.2 / 3$ \\
$\chi<-1 \& \alpha=1$ & & $\chi \lesssim-10$ \\
$\chi>-1 \& \alpha=2 / 3$ & $\frac{k}{2 \alpha^{2}|\beta|}\left[1-\left(1-\frac{2 \alpha^{2}|\beta|}{k}\right) e^{-\sqrt{\frac{2}{3 \alpha}}\left(\hat{\phi}-\hat{\phi}_{c}\right)}\right]$ & $\chi \gtrsim 2$ \\
\hline \hline
\end{tabular}

$\Psi \simeq \begin{cases}\frac{k}{2 \alpha^{2} \beta} C^{2-3 \alpha} e^{\sqrt{\frac{2}{3 \alpha}}(2-3 \alpha) \hat{\phi}}\left(C e^{\sqrt{\frac{2}{3 \alpha}} \hat{\phi}}-1\right) & (\chi<-1) \\ \frac{k}{-2 \alpha^{2} \beta} C^{2-3 \alpha} e^{-\sqrt{\frac{2}{3 \alpha}}(2-3 \alpha) \hat{\phi}}\left(1-C e^{-\sqrt{\frac{2}{3 \alpha}} \hat{\phi}}\right) & (\chi>-1)\end{cases}$

where $\beta \equiv-(1+\chi) / 6$ and $C$ is a positive constant. $C$ can be determined by, for example, $\Psi\left(\hat{\phi}_{c}\right)=1$. The results are summarized in Table I. For the cases of $\chi<-1 \& \alpha \neq 1$ and $\chi>-1 \& \alpha \neq 2 / 3,2 \alpha^{2}|\beta| / k \gg 1$ is further assumed, which will be discussed soon. The approximated expressions for $\Psi$ have similarity even for $\chi<-1$ and $\chi>-1$. Based on the expressions, we categorize the parameter space into two cases:

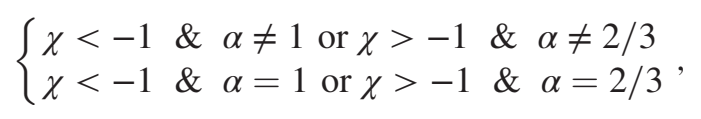

and we discuss the numerical results in Fig. 2.

$$
\text { 1. } \chi<-1 \& \alpha \neq 1 \text { or } \chi>-1 \& \alpha \neq 2 / 3
$$

As mentioned above, we further assume

$$
2 \alpha^{2}|\beta| / k \gg 1
$$

to derive the expressions, which are shown in the first row of Table I. If the above condition is satisfied, then the second term in the parenthesis of Eq. (53) can be ignored. (The condition can be rewritten as $|\beta| \phi_{c}^{2} \gg 1$.) We note here that this approximation is only valid for an $\alpha$ which is not much closer to 1 or $2 / 3$. Ignoring this term under the limit of $\alpha \rightarrow 1$ or $2 / 3$, one cannot determine a consistent $C$ from the analytic expressions.

To get a very rough picture, let us make more simplifications. If we further assume that the effective potential is determined by $V \sim g^{2} \xi^{2} \Psi$, then $n_{s}$ and $r$ are given by $\left(n_{s}, r\right) \sim\left(1-p^{2}, 8 p^{2}\right)$, where $p \equiv \sqrt{6 / \alpha}(1-\alpha)$ for $\chi<$ -1 and $p \equiv \sqrt{2 / 3 \alpha}(3 \alpha-2)$ for $\chi>-1$. The expressions indicate that for $\chi<-1(\chi>-1), n_{s}$ and $r$ get larger and smaller, respectively, when $\alpha$ approaches unity (2/3). This estimation is consistent with the results shown in Fig. 2. Although the qualitative behavior can be understood from this rough estimation, quantitative discussion is found to be more complicated.

For the $\chi<-1$ case, the approximation is found to be good for $\chi \lesssim-20$ and $\alpha \lesssim 2.8 / 3$. If $\alpha$ becomes larger, we find that the approximated expression of $\Psi$ does not give the correct values of $n_{s}$ and $r$ even though Eq. (56) is satisfied. The maximum values of $n_{s}$ and $r$ come from saturated values of $\phi_{*}$ in the limit $k \rightarrow 0$.

For the $\chi>-1$ case, on the other hand, the expression is found to be valid for $\chi \gtrsim-0.5$ and $\alpha \gtrsim 2.2 / 3$. However, we have found that the upper bound for $n_{s}$ (and $r$ ) is given by the stability of $\tau$. This is expected from Eq. (30). Therefore, it is difficult to understand the predicted value of $n_{s}$ and $r$ based on the simple approximation.

Therefore, to see the $\alpha$ dependence on $n_{s}$ and $r$ for cases of $\chi<-1$ and $\chi>-1$, it is more intuitive to plot the effective potential numerically. Figure 3 shows the effective potential $\hat{V}$ (normalized by the value at the critical point) plotted for various values of $\alpha$. Here we have derived $\hat{\phi}$ by numerically solving Eq. (45). One can see that $\hat{\phi}_{c}$ (and $\hat{\phi}_{*}$ ) becomes smaller as $\alpha$ approaches 1 . Consequently, a smaller $r$ is obtained, which is consistent with the results shown in Fig. 2.

\section{2. $\chi<-1 \& \alpha=1$ or $\chi>-1 \& \alpha=2 / 3$}

As seen in Table I, $\Psi$ is given in more complicated expressions compared to the previous case; meanwhile, both the $\alpha=1$ and $2 / 3$ cases give a similar $\Psi$ expression. This expression is valid when $\left|(1+\chi)^{2} \phi^{2} /\left(-2 \Phi_{0}\right)\right| \gg 1$ is satisfied. We have found that the approximated $\Psi$ is valid for $\chi \lesssim-10$ and $\chi \gtrsim 2$ when $\alpha=1$ and $2 / 3$, respectively. Besides this, recall that $k$ has a lower bound, given in Eq. (23). Aside from the exponential factor, $\Psi$ is given as a function of $2 \alpha^{2}|\beta| / k$, and it has the same upper bound:

$$
2 \alpha^{2}|\beta| / k<1 \text {. }
$$

Therefore, the predicted values of $n_{s}$ and $r$ for $\alpha=1$ and $2 / 3$ are expected to be similar to each other. ${ }^{11}$ In fact, Fig. 2 shows the expected results. We have found that the lowest

\footnotetext{
${ }^{11}$ The model with $\alpha=1$ is the same model studied in Ref. [33]. The predictions for $\left(n_{s}, r\right)$ are different from those in the literature, because now we consider $\chi$ away from -1 .
} 

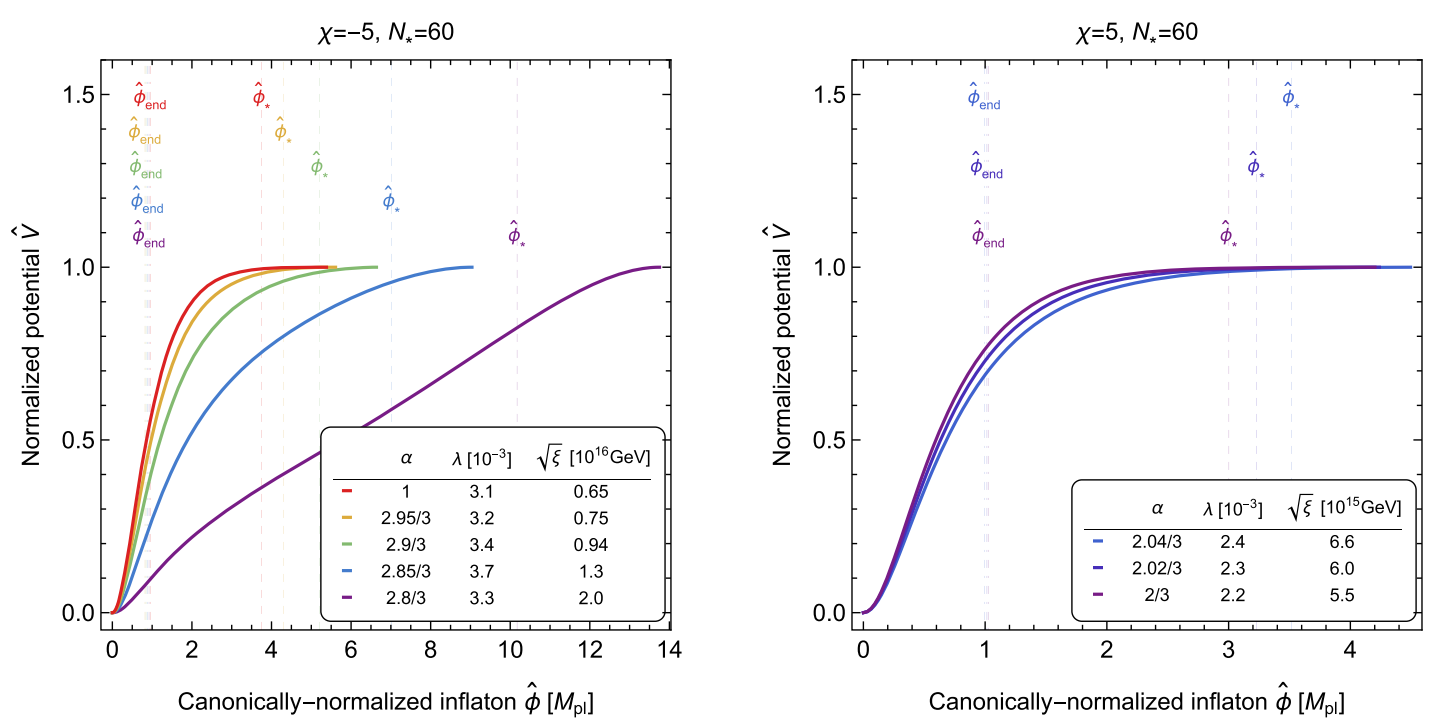

FIG. 3. Normalized potential $\hat{V}$ as a function of the canonically normalized inflaton field $\hat{\phi}$. Only the subcritical regime is shown, and the field values of $\hat{\phi}$ at the end of inflation and at $60 e$-folds are indicated as $\hat{\phi}_{\text {end }}$ and $\hat{\phi}_{*}$, respectively. Color codes are the same as in Fig. 2, and $\lambda$ and $\sqrt{\xi}$ are chosen to give the best-fit value of the observed $n_{s}$.
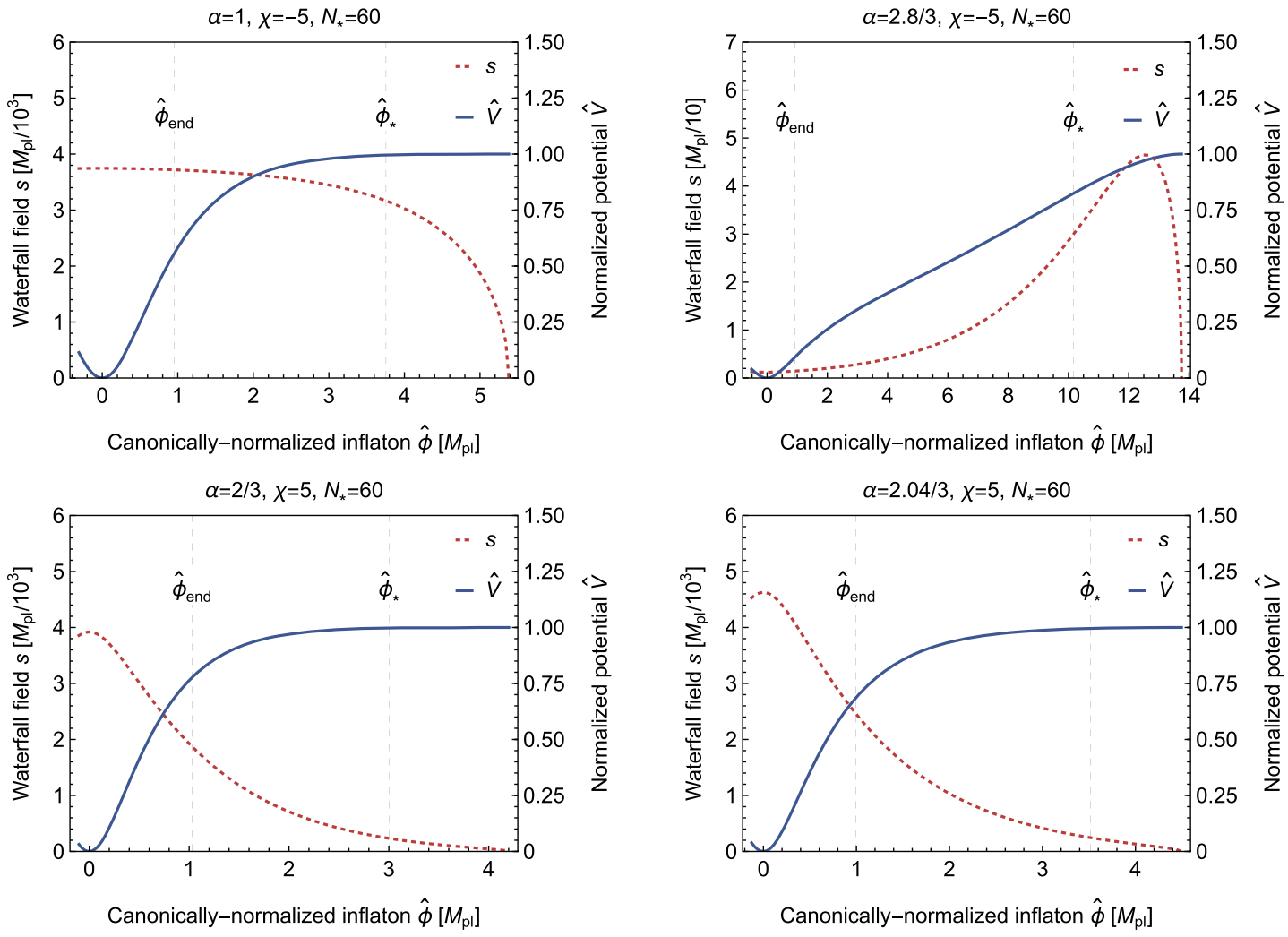

FIG. 4. Trajectory of the waterfall field (red dotted curve) as a function of the canonically normalized inflaton field $\hat{\phi}$. As a reference, the normalized potential $\hat{V}$ (blue solid curve) is also plotted. The field values of $\hat{\phi}$ at the end of inflation and at $60 e$-folds are indicated as $\hat{\phi}_{\text {end }}$ and $\hat{\phi}_{*}$, respectively. In the top (bottom) panels, $\chi$ is taken to -5 (5). The other parameters are $\alpha=1, \lambda=3.1 \times 10^{-3}, \sqrt{\xi}=$ $6.5 \times 10^{15} \mathrm{GeV} \quad$ (top-left); $\quad \alpha=2.8 / 3, \quad \lambda=3.3 \times 10^{-3}, \quad \sqrt{\xi}=2.0 \times 10^{16} \mathrm{GeV} \quad$ (top-right); $\quad \alpha=2 / 3, \quad \lambda=2.2 \times 10^{-3}, \quad \sqrt{\xi}=$ $5.5 \times 10^{15} \mathrm{GeV}$ (bottom-left); and $\alpha=2.04 / 3, \lambda=2.4 \times 10^{-3}, \sqrt{\xi}=6.6 \times 10^{15} \mathrm{GeV}$ (bottom-right). With these parameters, the median value of the observed $n_{s}$ is obtained, and the tensor-to-scalar ratio is predicted to be $7.9 \times 10^{-4}$ (top-left), $6.4 \times 10^{-2}$ (top-right), $4.2 \times 10^{-4}$ (bottom-left), and $8.5 \times 10^{-4}$ (bottom-right). 
value of $k$ determines the maximum values for $n_{s}$ and $r$, and that the results resemble each other. One can see this fact from the plot of the effective potential shown in Fig. 3. We will come back to $\alpha=1$ and $2 / 3$ cases with various values of $\chi$ in Sec. IV D.

In a nutshell, for $|\chi| \gg 1$, the potential becomes flatter as $\alpha$ approaches unity or $2 / 3$. This allows $\hat{\phi}_{*}$ to have smaller values, and consequently, we obtain smaller $r$ values.

Finally, we plot the trajectory of the waterfall field as a function of $\hat{\phi}$ in Fig. 4. The potential is found to be almost flat at $60 e$-folds from the end of inflation except for the $\alpha=2.8 / 3$ and $\chi=-5$ case. In this case, the waterfall field grows much larger than the global minimum value due to a factor

$-\frac{\Phi_{0}}{3}(1-\Psi) \sim-\left(\frac{2 \alpha^{2} \beta}{k}\right)^{\frac{1}{3(1-\alpha)}} \sqrt{\frac{6}{\alpha}}(1-\alpha)\left(\hat{\phi}-\hat{\phi}_{c}\right)$,
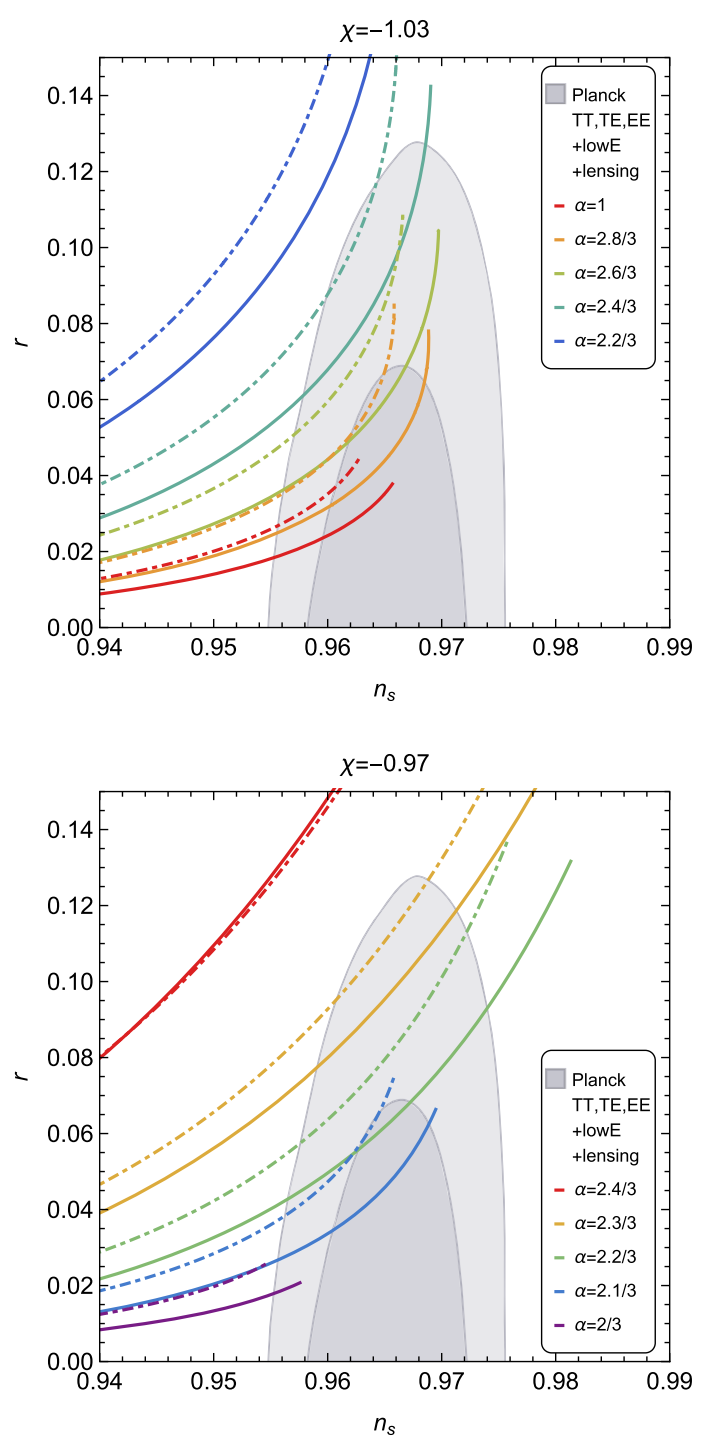

where $\left(2 \alpha^{2} \beta / k\right)^{1 / 3(1-\alpha)} \sim \mathcal{O}\left(10^{5}\right)$ after the critical point. Due to this enhancement, the effective potential is distorted to have convection points. It is worth noticing that the subcritical regime of the superconformal hybrid inflation reduces to such a potential and that predicted $n_{s}$ and $r$ values can be consistent with the observations. In the other cases, on the other hand, there is no such enhancement after the critical point, and the waterfall field grows slowly as shown in the figure.

$$
\text { C. } \chi \simeq-1
$$

Summary of the predictions.-Figure 5 is the same as Fig. 2, but taking $\chi=-1.03$ (top) and $\chi=-0.97$ (bottom) and various values of $\alpha$. In the right panels, the results are given for $N_{*}=60$ and 55 (since the allowed region for $N_{*}=50$ is very limited). As in the case of $|\chi| \gtrsim 5, n_{s}$
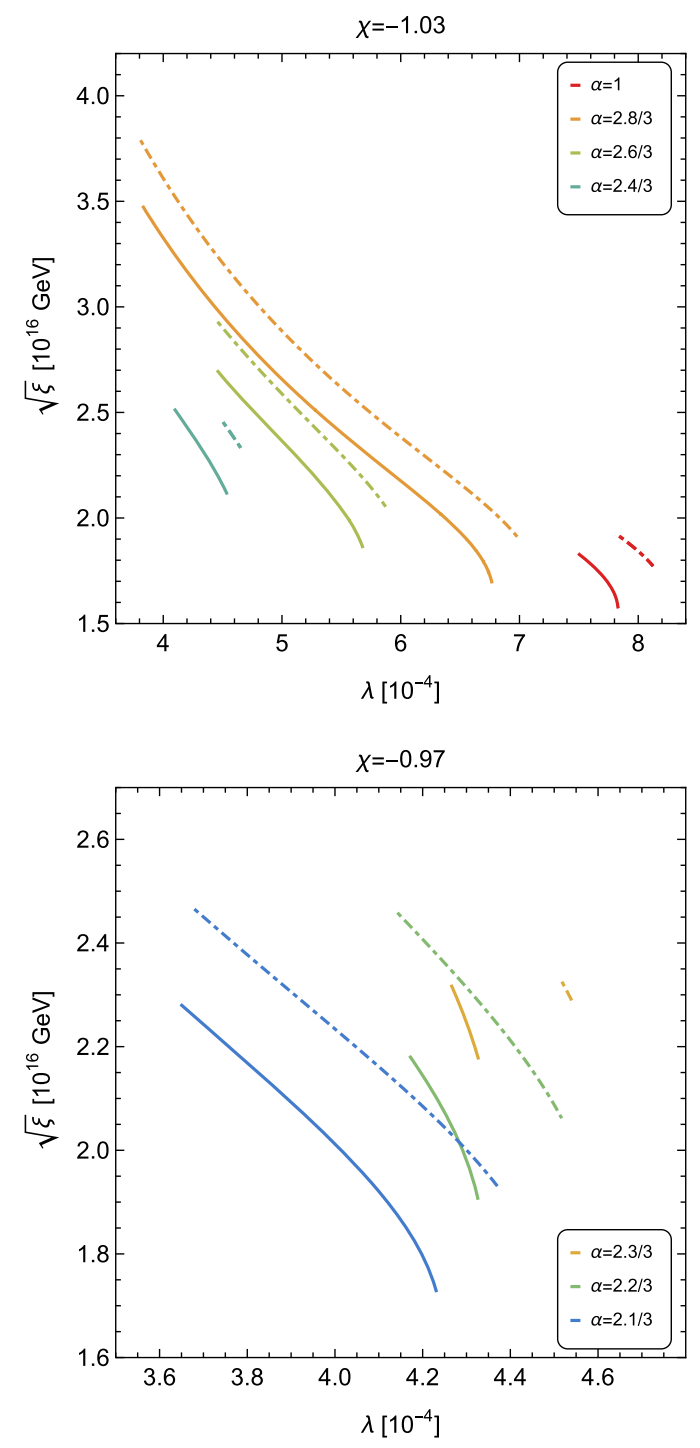

FIG. 5. Same as Fig. 2, but taking $\chi=-1.03$ (top) and $\chi=-0.97$ (bottom) and different values of $\alpha$ accordingly. In the right panels, we take $N_{*}=60$ (solid curves) and $N_{*}=55$ (dot-dashed curves). 
and $r$ become larger for smaller $k$. It is found that $10^{-2} \lesssim$ $r \lesssim 0.1$ for both cases in the region consistent with the Planck data. While the result with $\chi=-1.03$ is similar to one given in Ref. [33], $r$ becomes larger for smaller $\alpha$, and it is eventually out of the preferred region by the Planck observations. The results of $\chi=-0.97$ look similar. However, $r$ becomes smaller for smaller $\alpha$. The allowed region is found to be $10^{-4} \lesssim \lambda \lesssim 10^{-3}$ and $\sqrt{\xi} \sim$ $\mathcal{O}\left(10^{16} \mathrm{GeV}\right)$ for both cases.

As in the previous subsection, we derive the effective potential in terms of the canonically normalized field. In the present case, the second term in the parenthesis on the rhs in Eq. (46) can be ignored due to $\chi+$ $1 \simeq 0$ to get

$$
K_{N \bar{N}} \simeq-\frac{3 \alpha}{\Phi_{0}}
$$

Consequently, $\phi$ and $\Phi_{0}$ are given by

$$
\begin{gathered}
\phi^{2} \simeq \begin{cases}\frac{1}{\beta} \sinh ^{2} \sqrt{\frac{\beta}{\alpha}} \hat{\phi} & (\chi<-1) \\
\frac{1}{-\beta} \sin ^{2} \sqrt{\frac{-\beta}{\alpha}} \hat{\phi} & (\chi>-1)\end{cases} \\
-\frac{\Phi_{0}}{3} \simeq \begin{cases}\cosh ^{2} \sqrt{\frac{\beta}{\alpha}} \hat{\phi} & (\chi<-1) \\
\cos ^{2} \sqrt{\frac{-\beta}{\alpha}} \hat{\phi} & (\chi>-1)\end{cases}
\end{gathered}
$$

and $\Psi$ is given in Table II. Here we have taken the boundary condition $\hat{\phi}=0$ at $\phi=0$. One can see that both cases give similar expressions. In fact, both are exactly the same around $\phi=0$, and the qualitative difference appears at large field values. ${ }^{12}$ We find that the approximated expression is valid in $|1+\chi| \lesssim 0.05$ and $|1+\chi| \lesssim 0.01$ for $\chi<-1$ and $\chi>-1$, respectively. Since the valid parameter region is limited, it is better to see $\alpha$ dependence numerically by computing the potential, which is shown in Fig. 6. From the figure, one can see that the potential becomes flatter as $\alpha$ approaches $1(2 / 3)$ and gives smaller values of $r$ for $\chi=-1.03(-0.97)$. This behavior is similar to that seen in Sec. IV B, and it can be understood qualitatively as follows. Expanding the effective potential in terms of $\hat{\phi}$, the tensor-to-scalar ratio is approximately given by

$$
r \sim \frac{32}{\hat{\phi}_{*}^{2}}+\frac{32(9 \alpha-7)(1+\chi)}{9 \alpha} .
$$

\footnotetext{
${ }^{12}$ As mentioned earlier, the $\chi=-1$ case corresponds to the model studied in Refs. [7,8] when potential is written in terms of $\hat{\phi}$.
}

TABLE II. Approximated expression of $\Psi$ in the limit $\left|(1+\chi)^{2} \phi^{2} /\left(-2 \Phi_{0}\right)\right| \ll 1$. The parameter space where the approximated expression is valid is given in the right column. The effective potential can be obtained from $\Psi$ by using Eq. (42).

\begin{tabular}{lll}
\hline \hline Cases & Approximated expression for $\Psi$ & Valid parameters \\
\hline$\chi<-1$ & $\frac{k}{2 \alpha^{2} \beta} \cosh ^{2(2-3 \alpha)} \sqrt{\frac{\beta}{\alpha}} \hat{\phi} \times \sinh ^{2} \sqrt{\frac{\beta}{\alpha}} \hat{\phi}$ & $|1+\chi| \lesssim 0.05$ \\
$\chi>-1$ & $\frac{k}{2 \alpha^{2}|\beta|} \cos ^{2(2-3 \alpha)} \sqrt{\frac{\mid \beta}{\alpha}} \hat{\phi} \times \sin ^{2} \sqrt{\frac{\mid \beta}{\alpha}} \hat{\phi}$ & $|1+\chi| \lesssim 0.01$ \\
\hline \hline
\end{tabular}

Due to the second term, $r$ becomes smaller (larger) as $\alpha$ gets larger when $\chi<-1 \quad(\chi>-1)$. We note that the above rough estimate cannot give the precise value for $r$; however, it is enough to understand the response to the value of $\alpha$. In addition, we find that $\alpha=2 / 3$ is not allowed for $\chi=-0.97$. This comes from the lower bound for $k$ given in Eq. (23). We will discuss this in the next subsection in detail by comparing the results with $\alpha=1$. Except for $\alpha=2 / 3$ or 1 , we find that the endpoint of $n_{s}$ and $r$ comes from the saturated value of $\phi_{*}$ under $k \rightarrow 0$.

For comparison with the $|\chi| \gtrsim 5$ case, Fig. 7 shows the trajectory of the waterfall field. Although the potential is not so flat, a large field value keeps the inflaton slow-roll. For $\chi<-1$, the waterfall field grows to its global minimum value just after entering the subcritical regime, but it does not overshoot much greater than the global minimum.

\section{Specific cases}

Finally, we focus on the specific cases where $\alpha=1$, $\alpha=2 / 3$, and $\chi=0$. Such values of the parameters are motivated by superstring theory or superconformal symmetry. Therefore, it is worth analyzing these cases in detail, although parts of the results shown in this subsection are already presented in the previous subsections. As is found in the previous subsections, the results for $\alpha=1$ and $2 / 3$ share some behaviors. Thus, we categorize the contents as $\alpha=1$ or $2 / 3$, and $\chi=0$.

\section{1. $\alpha=1$ or $2 / 3$}

Summary of the predictions.-Figure 8 shows the predicted $n_{s}$ and $r$ (left panels) and the allowed region (right panels) for $\alpha=1$ (top) and $\alpha=2 / 3$ (bottom) and various values of $\chi$. The allowed parameters are roughly $\lambda \sim$ $\mathcal{O}\left(10^{-3}\right)$ and $\sqrt{\xi} \sim \mathcal{O}\left(10^{16} \mathrm{GeV}\right)$ and mildly depend on $\chi$. For $\alpha=1, \chi<-1$ gives consistent results with the Planck observations for $N_{*}=60$, while $N_{*}=50$ is found to be disfavored. For $\alpha=2 / 3, n_{s}$ and $r$ are consistent with the observed data when $\chi \gtrsim 0$ or $\chi \simeq-1$. In the latter case, $r \sim 0.1$, and $N_{*}=60$ is preferred. As in the $\alpha=1$ case, $N_{*}=50$ has a tension with the observed data. 

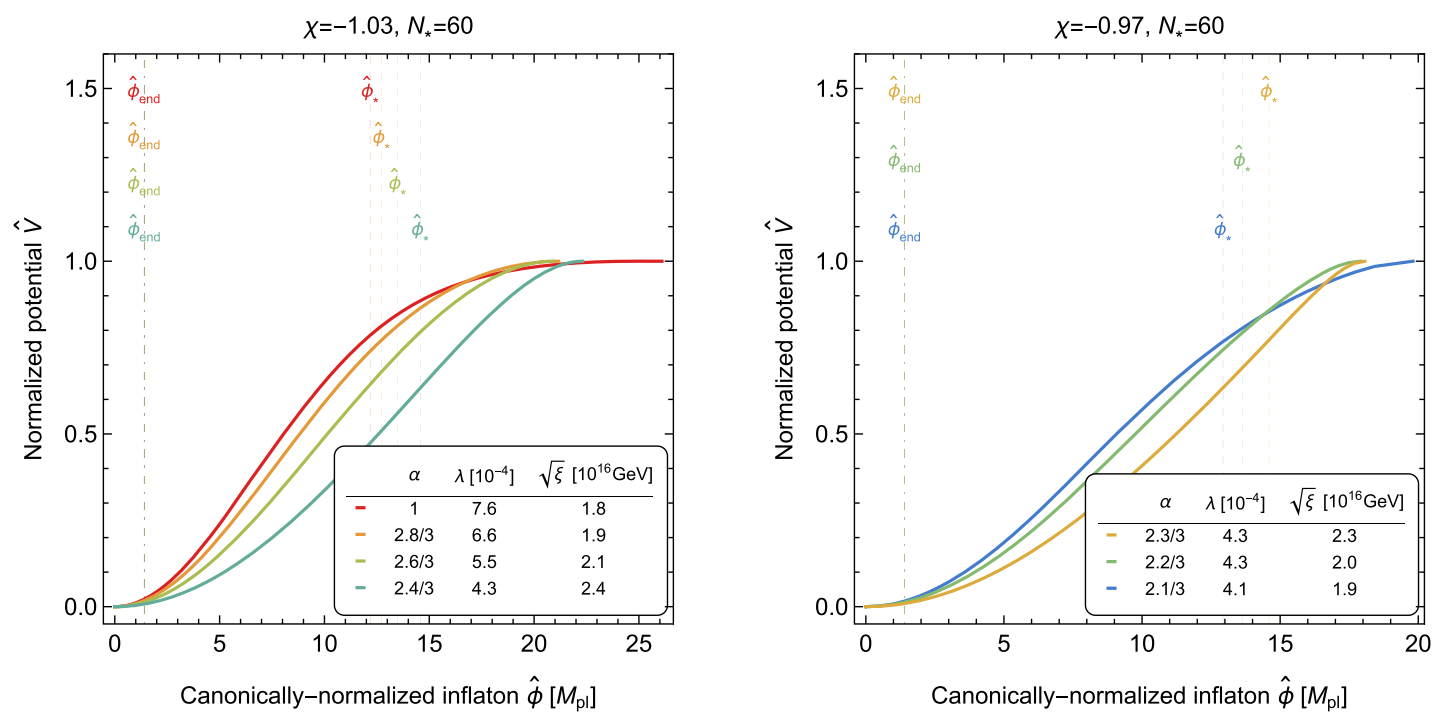

FIG. 6. Same as Fig. 3, but with $\chi=-1.03$ and -0.97 . Color codes are the same as in Fig. 5, and $\lambda$ and $\sqrt{\xi}$ are chosen to give the bestfit value for $n_{s}$.
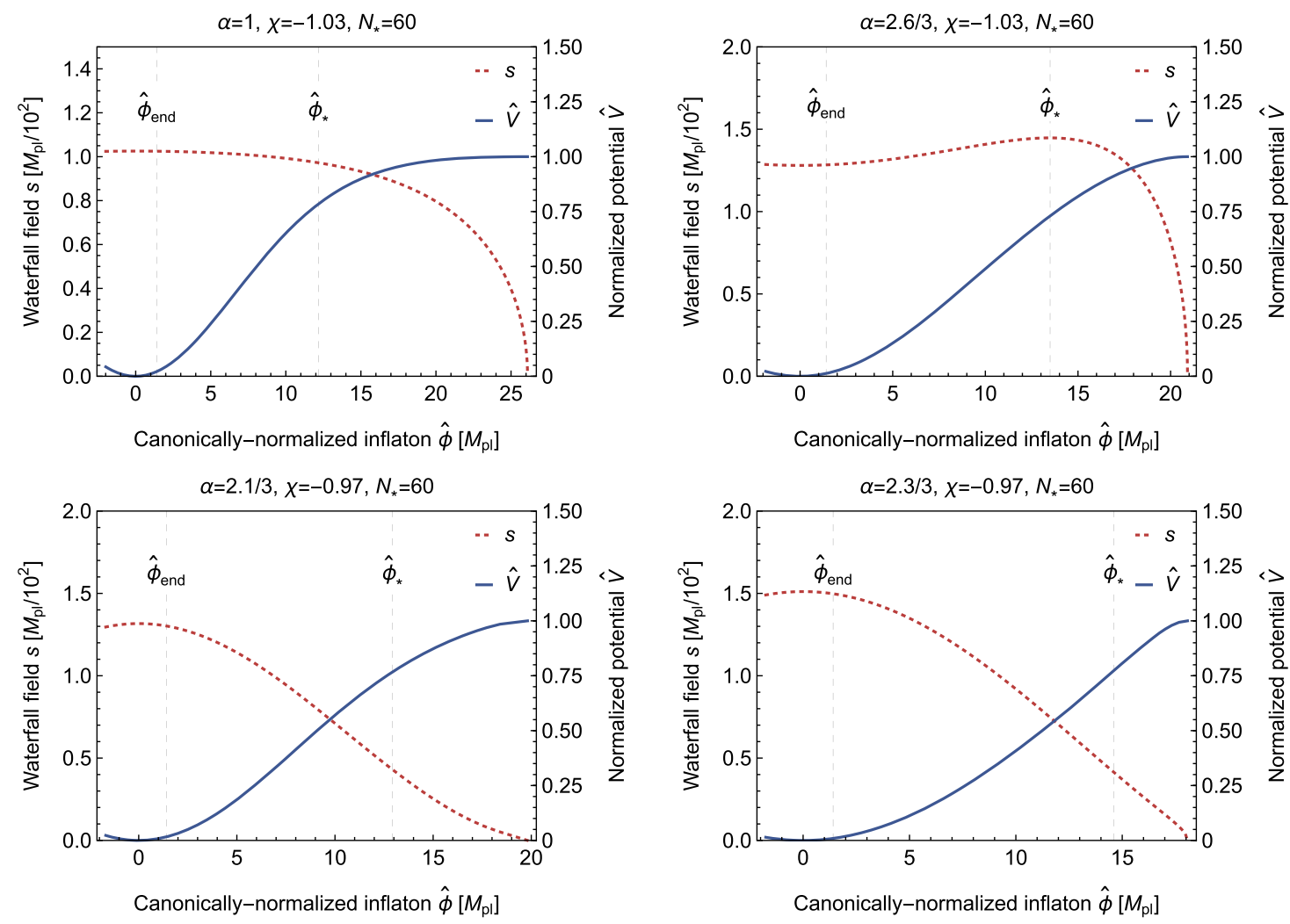

FIG. 7. Same as Fig. 4, but taking $\chi=-1.03$ (top) and $\chi=-0.97$ (bottom). The other parameters in each panel are $\alpha=1$, $\lambda=7.6 \times 10^{-4}, \quad \sqrt{\xi}=1.8 \times 10^{16} \mathrm{GeV} \quad$ (top-left); $\quad \alpha=2.6 / 3, \quad \lambda=5.5 \times 10^{-4}, \quad \sqrt{\xi}=2.1 \times 10^{16} \mathrm{GeV}$ (top-right); $\quad \alpha=2.1 / 3$, $\lambda=4.1 \times 10^{-4}, \sqrt{\xi}=1.9 \times 10^{16} \mathrm{GeV}$ (bottom-left); and $\alpha=2.3 / 3, \lambda=4.3 \times 10^{-4}, \sqrt{\xi}=2.3 \times 10^{16} \mathrm{GeV}$ (bottom-right). The tensor-to-scalar ratio is found to be $3.5 \times 10^{-2}$ (top-left), $5.9 \times 10^{-2}$ (top-right), $4.5 \times 10^{-2}$ (bottom-left), and $r=9.5 \times 10^{-2}$ (bottom-right). 

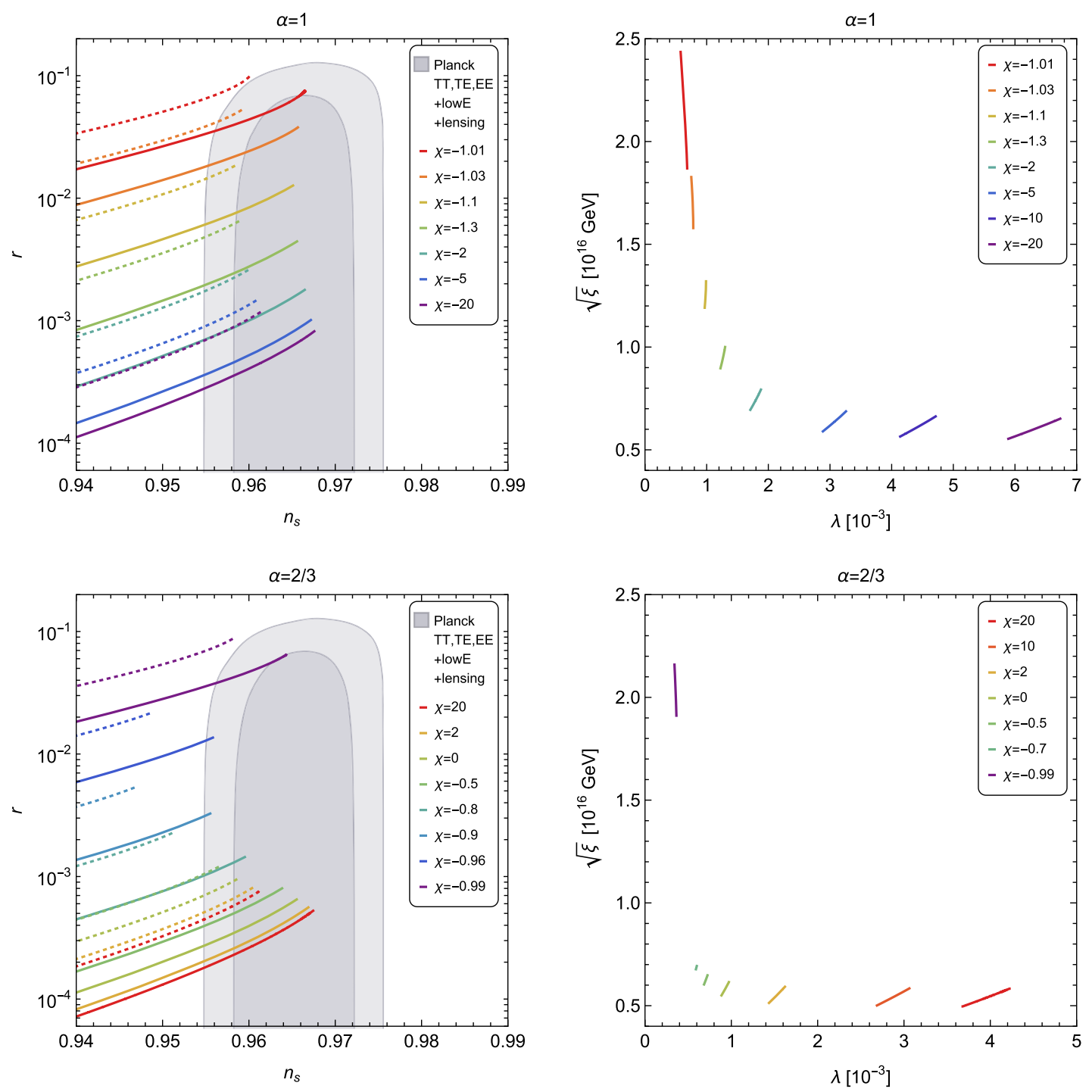

FIG. 8. Left: Predicted values of $n_{s}$ and $r$ for $N_{*}=60$ (solid curves) and 50 (dotted curves) and various values of $\chi$. $\alpha$ is taken to be 1 (top) and 2/3 (bottom). Right: Allowed parameters for $N_{*}=60$. Here, $\alpha=1$ (top) and 2/3 (bottom), and various values of $\chi$ are taken.

It can be seen the resultant $n_{s}$ and $r$ for $\alpha=1$ and $2 / 3$ are similar when $\chi \simeq-1$ and $|\chi| \gtrsim 5$. The similarity at $\chi \simeq-1$ is due to the fact that the effective potential reduces to the same potential as shown in Table II. In the $|\chi| \gtrsim 5$ case, $\Psi$ has the same form of function but with a different exponent of the exponential factor (see Table I) and the valid domain of the parameter. That is why the behavior is quite similar. However, they give quantitatively different predictions for $n_{s}$ and $r$, which comes from the different exponent in $\Psi$. The quantitative difference is prominent in the other value of $\chi$ : i.e., $\chi \lesssim-1$ and $\chi \gtrsim-1$.
Figure 9 shows $\chi$ dependence on the potential. It is clearly seen that the potential gets flatter for larger values of $|\chi|$ for both $\alpha=1$ and $2 / 3$, and consequently, $r$ is suppressed.

$$
\text { 2. } \chi=0
$$

Summary of the predictions.-Figure 10 shows the $n_{s}, r$, and the allowed parameter space for $\chi=0$. Roughly speaking, the results are found to be similar to those in the $\chi \gtrsim 5$ case (see Fig. 2). It is found that $\alpha \lesssim 2.16 / 3$ gives 

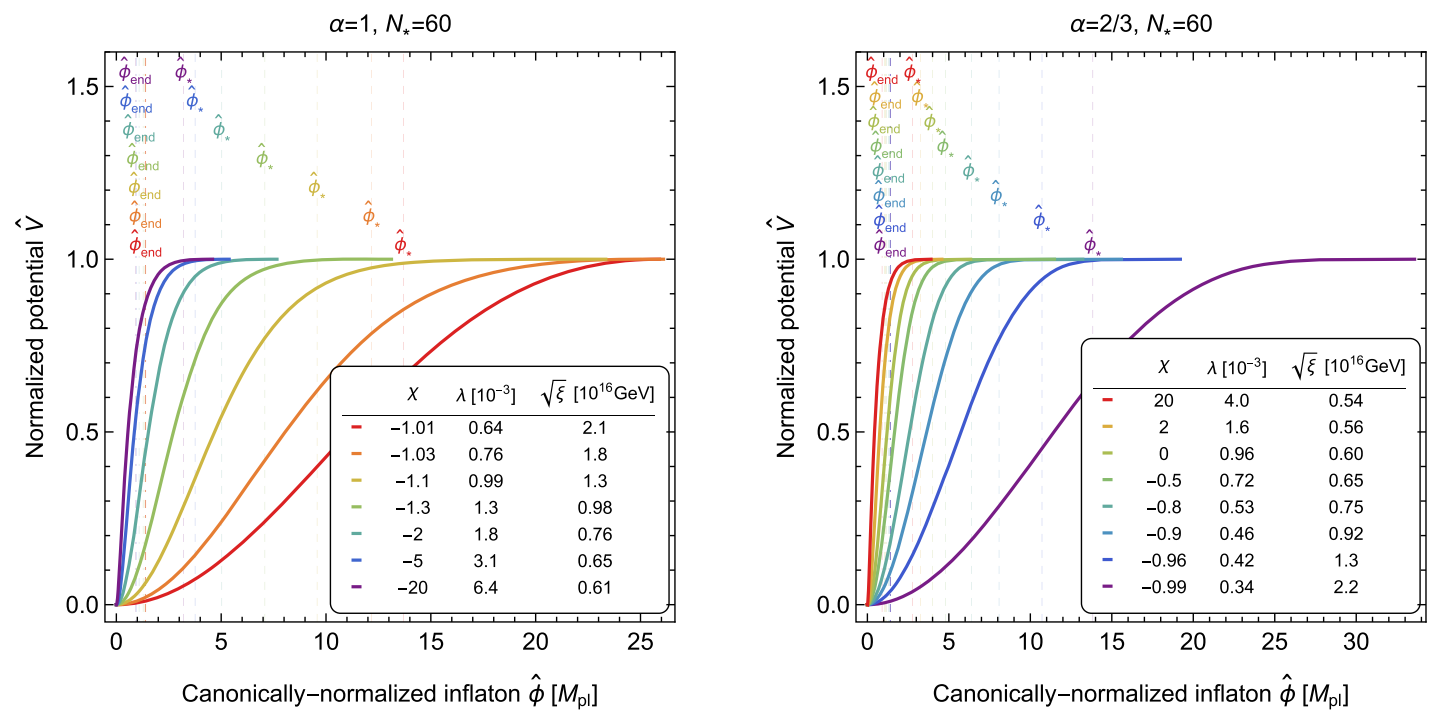

FIG. 9. Normalized potential as a function of the canonically normalized inflaton field. Color codes are the same as in Fig. 8, and $\lambda$ and $\sqrt{\xi}$ are chosen to give the best-fit value for $n_{s}$.
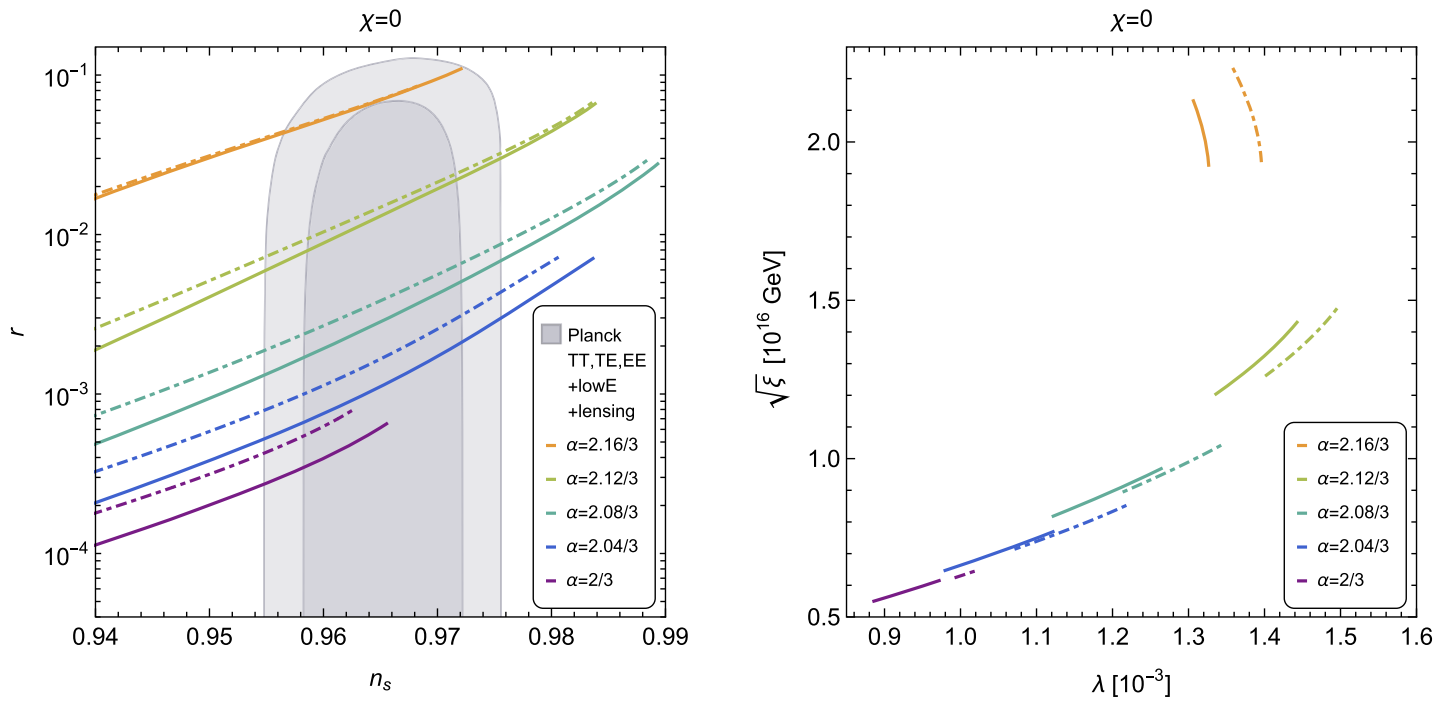

FIG. 10. Same as Fig. 2, but taking $\chi=0$ and different values of $\alpha$ accordingly. In the right panels, we take $N_{*}=60$ (solid curves) and $N_{*}=55$ (dot-dashed curves).

a consistent result with the observations for both $N_{*}=60$ and 50. A notable difference is that $r$ can be as large as $\mathcal{O}\left(10^{-2}\right)$.

In the $\chi=0$ case, it is easy to derive the potential analytically, since the Kähler metric [Eq. (46)] takes a simple form without any approximation:

$$
K_{N \bar{N}}=\frac{36 \alpha}{\left(6-\phi^{2}\right)^{2}}
$$

As a result, Eq. (45) can be solved analytically to give

$$
\begin{gathered}
\phi^{2}=6 \tanh ^{2} \frac{\hat{\phi}}{\sqrt{6 \alpha}}, \\
-\frac{\Phi_{0}}{3}=\cosh ^{-2} \frac{\hat{\phi}}{\sqrt{6 \alpha}}, \\
\Psi=\frac{3 k}{\alpha^{2}} \tanh ^{2} \frac{\hat{\phi}}{\sqrt{6 \alpha}} \times \cosh ^{2(3 \alpha-2)} \frac{\hat{\phi}}{\sqrt{6 \alpha}} .
\end{gathered}
$$

Here, we have taken the boundary condition $\hat{\phi}=0$ at $\phi=0$. Although the effective potential is given exactly, it behaves nontrivially as a function of $\alpha$. When $\alpha$ is away 


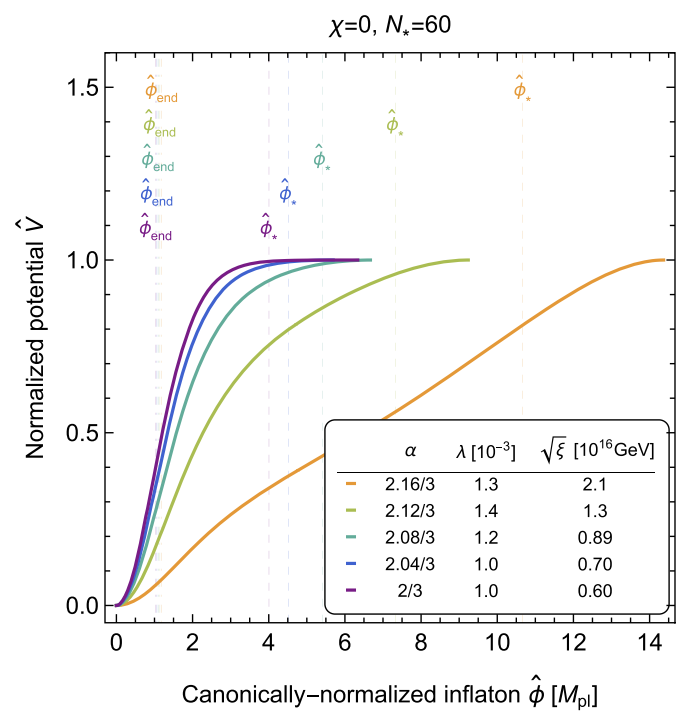

FIG. 11. Same as Fig. 3, but with $\chi=0$. Color codes are the same as in Fig. 10, and $\lambda$ and $\sqrt{\xi}$ are chosen to give the best-fit value for $n_{s}$.

from $2 / 3$, the discussion for $\chi \gtrsim 5$ case in Sec. IV B can be applied. Namely, when $\alpha$ gets smaller, $r$ becomes smaller. If $\alpha$ goes much closer to $2 / 3$, on the other hand, Eq. (56) is no longer satisfied. Instead, we can derive the effective potential for $\alpha=2 / 3$ as

$$
V=\frac{27 g^{2} \xi^{2} k}{4} \tanh ^{2} \frac{\hat{\phi}}{2} \times\left(1-\frac{27 k}{8} \tanh ^{2} \frac{\hat{\phi}}{2}\right)
$$

The largest values of $n_{s}$ and $r$ are obtained from the lower bound for $k$. The resultant $n_{s}$ and $r$ that are consistent with the observed data are found for $N_{*}=60$ and 50. In order to see $\alpha$ dependence on $n_{s}$ and $r$, it is more intuitive to plot the effective potential, which is shown in Fig. 11. As $\alpha$ approaches $2 / 3$, it can be seen that the potential becomes flatter, and smaller values of $r$ are obtained.

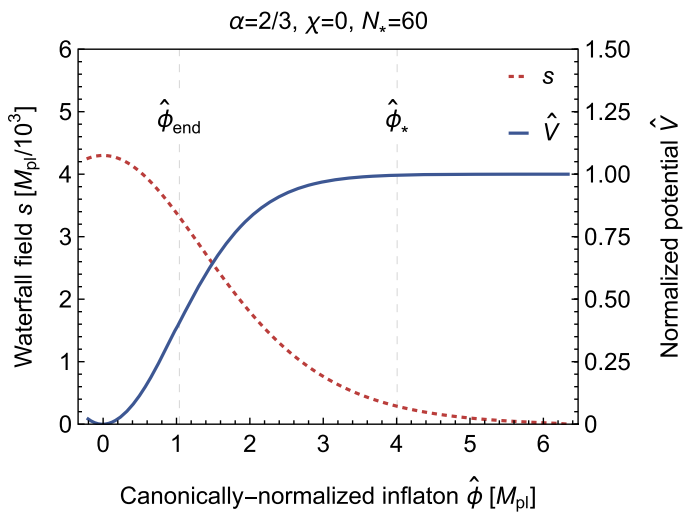

For completeness, Fig. 12 shows the same plot as Fig. 4 but takes $\chi=0$ and $\alpha=1,2.16 / 3$. It can be seen that the shape of the potential changes nontrivially depending on $\alpha$. That is why the prediction for $r$ changes by orders of magnitude for an $\alpha$ that even slightly deviates from $2 / 3$.

\section{CONCLUSIONS AND DISCUSSION}

We have studied the subcritical regime of $D$-term hybrid inflation in the generalized framework of a superconformal model. The model is characterized by the superconformal Kähler potential and superconformal superpotential. The former contains a parameter $\alpha$ and an explicit superconformal breaking term that is turned on by nonzero $\chi$. The latter is given by the Yukawa interaction of the inflaton and waterfall fields with a coupling $\lambda$. In addition, we introduce the Fayet-Iliopoulos term $\xi$ that appears after gauge fixing of the superconformal symmetry. In this framework, we focus on the parameter space $\lambda \ll 1$, which is supported by an approximate shift symmetry of the inflaton field, and $\alpha \leq 1(\alpha \geq 2 / 3)$ for $\chi<-1(\chi>-1)$ to give a single critical point.

In the parameter space, it has been found that inflation continues in the subcritical regime of the inflaton field, and that the inflaton potential in the subcritical regime changes drastically depending on $\alpha$ and $\chi$. The latest Planck data prefer $2 / 3 \leq \alpha \leq 1$ for $\chi \simeq-1$ and $\alpha \simeq 1(2 / 3)$ for $\chi \lesssim-1$ $(\chi \gtrsim-1)$ for $60 e$-folds, while the preferred parameter space is limited for $50 e$-folds as $2.5 / 3 \lesssim \alpha \leq 1(2 / 3 \leq$ $\alpha \lesssim 2.2 / 3)$ for $\chi<-1(\chi>-1)$. The tensor-to-scalar ratio $r$ turns out to be $r>\mathcal{O}\left(10^{-2}\right)$ for $\chi \simeq-1, r>\mathcal{O}\left(10^{-3}\right)$ for $\chi \lesssim-1$, and $r \sim \mathcal{O}\left(10^{-4}\right)$ for $\chi \gtrsim-1$ for $60 e$-folds. Roughly speaking, $r$ tends to be suppressed when $\alpha$ approaches 1 for $\chi<-1$, or $2 / 3$ for $\chi>-1$ or $|\chi| \gg 1$.

The other parameters, $\lambda$ and $\xi$, on the other hand, are $\mathcal{O}\left(10^{-4}\right)<\lambda<\mathcal{O}\left(10^{-2}\right)$ and $\sqrt{\xi} \sim \mathcal{O}\left(10^{16} \mathrm{GeV}\right)$. This result indicates that the FI term is determined to be around the GUT scale, which might be a clue for further

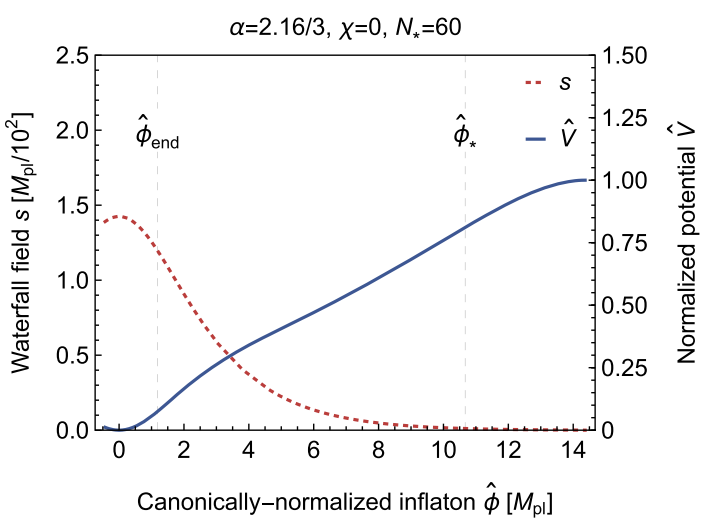

FIG. 12. Same as Fig. 4, but taking $\chi=0$ and $\alpha=2 / 3$ (left) and $2.16 / 3$ (right). In the left panel, $\lambda=9.6 \times 10^{-4}$ and $\sqrt{\xi}=6.0 \times 10^{15} \mathrm{GeV}$. In the right panel, $\lambda=1.3 \times 10^{-3}$ and $\sqrt{\xi}=2.1 \times 10^{16} \mathrm{GeV}$. The tensor-to-scalar ratio is $6.1 \times 10^{-4}$ (left) and $7.0 \times 10^{-2}$ (right). 
phenomenological study of the GUT, neutrino sector, and inflation [15,38-40].

Besides this, the cases of integer $3 \alpha$ (namely, 2 or 3 in the present model) and $\chi=0$ are motivated by the compactification of the extra dimensions in superstring theory and the superconformal symmetry, respectively. We have found the allowed parameter spaces for such cases. This may bring another clue to investigating the relation between the symmetry of the compactified space and extension of the minimal supersymmetric standard model that accommodates the inflaton sector.

As mentioned in the Introduction, non-Abelian discrete symmetry can be one of such symmetries. Recently, the modular symmetry has drawn a lot of attention [41] to giving a nice fit with the experimental results of neutrino oscillations-for example, under the modular $S_{3}$ [42], $A_{4}$ [41-50], $S_{4}$ [51-53], and $A_{5}$ [54,55]. Furthermore, the study of the modular symmetry has been applied to solve the cosmological issues. Reference [56] has studied leptogenesis with the modular $A_{4}$ and showed that right-handed neutrinos with a mass scale of $10^{13} \mathrm{GeV}$ can account for the observed baryon asymmetry of the Universe. This coincides with the mass scale of right-handed sneutrinos that play the role of inflaton and can be a source of baryon asymmetry in the superconformal framework embedded into the MSSM [15,33]. Since the model proposed in
Ref. [15] predicts that one of the light neutrinos is massless, the model may give completely different consequences on inflation and the leptogenesis if the modular symmetry rules the lepton sector. Additionally, it was pointed out that higher-dimension operators that are allowed by the modular symmetry in the Kähler potential have the possibility to spoil the success of fitting with the neutrino oscillation data [57]. To avoid such a danger, a large volume limit in superstring theory $[58,59]$ is considered. For instance, the model with an additional gauge singlet Higgs in the large volume limit can give a consistent result with the neutrino oscillation data [60]. However, whether it is compatible with the cosmological issues needs further investigation. Our results, especially on the symmetry-enhanced points, would be another possibility to provide a phenomenologically and cosmologically acceptable scenario [61].

\section{ACKNOWLEDGMENTS}

We are grateful to Tatsuo Kobayashi and Tomo Takahashi for valuable discussions. We also thank Takashi Shimomura for hosting the Miyazaki Workshop on Particle Physics and Cosmology 2020, where this work was initiated. This work was supported by JSPS KAKENHI Grants No. JP17K14278, No. JP17H02875, No. JP18H05542, and No. JP20H01894 (K. I.).
[1] N. Aghanim et al. (Planck Collaboration), Astron. Astrophys. 641, A6 (2020).

[2] Y. Akrami et al. (Planck Collaboration), Astron. Astrophys. 641, A10 (2020).

[3] A. A. Starobinsky, Phys. Lett. 91B, 99 (1980).

[4] V. F. Mukhanov and G. V. Chibisov, JETP Lett. 33, 532 (1981), https://inspirehep.net/literature/170051.

[5] R. Kallosh, A. Linde, and D. Roest, J. High Energy Phys. 11 (2013) 198.

[6] W. Buchmuller, V. Domcke, and K. Kamada, Phys. Lett. B 726, 467 (2013).

[7] W. Buchmuller, V. Domcke, and K. Schmitz, J. Cosmol. Astropart. Phys. 11 (2014) 006.

[8] W. Buchmuller and K. Ishiwata, Phys. Rev. D 91, 081302 (2015).

[9] S. Clesse, Phys. Rev. D 83, 063518 (2011).

[10] H. Kodama, K. Kohri, and K. Nakayama, Prog. Theor. Phys. 126, 331 (2011).

[11] S. Clesse and B. Garbrecht, Phys. Rev. D 86, 023525 (2012).

[12] K. Freese, J. A. Frieman, and A. V. Olinto, Phys. Rev. Lett. 65, 3233 (1990).

[13] Y. Mikura, Y. Tada, and S. Yokoyama, Europhys. Lett. 132, 39001 (2020).
[14] Y. Mikura, Y. Tada, and S. Yokoyama, Phys. Rev. D 103, L101303 (2021).

[15] Y. Gunji and K. Ishiwata, J. High Energy Phys. 09 (2019) 065.

[16] P. Adamson et al. (MINOS Collaboration), Phys. Rev. Lett. 110, 251801 (2013).

[17] P. Adamson et al. (MINOS Collaboration), Phys. Rev. Lett. 110, 171801 (2013).

[18] K. Abe et al. (T2K Collaboration), Phys. Rev. D 96, 092006 (2017); 98, 019902(E) (2018).

[19] K. Abe et al. (T2K Collaboration), Phys. Rev. Lett. 121, 171802 (2018).

[20] P. Adamson et al. (NOvA Collaboration), Phys. Rev. Lett. 118, 231801 (2017).

[21] M. A. Acero et al. (NOvA Collaboration), Phys. Rev. D 98, 032012 (2018).

[22] S. Vagnozzi, E. Giusarma, O. Mena, K. Freese, M. Gerbino, S. Ho, and M. Lattanzi, Phys. Rev. D 96, 123503 (2017).

[23] E. Ma and G. Rajasekaran, Phys. Rev. D 64, 113012 (2001).

[24] K. S. Babu, E. Ma, and J. W. F. Valle, Phys. Lett. B 552, 207 (2003).

[25] G. Altarelli and F. Feruglio, Nucl. Phys. B720, 64 (2005).

[26] G. Altarelli and F. Feruglio, Rev. Mod. Phys. 82, 2701 (2010). 
[27] H. Ishimori, T. Kobayashi, H. Ohki, Y. Shimizu, H. Okada, and M. Tanimoto, Prog. Theor. Phys. Suppl. 183, 1 (2010).

[28] S. F. King and C. Luhn, Rep. Prog. Phys. 76, 056201 (2013).

[29] S. F. King, A. Merle, S. Morisi, Y. Shimizu, and M. Tanimoto, New J. Phys. 16, 045018 (2014).

[30] S. Ferrara, R. Kallosh, A. Linde, A. Marrani, and A. Van Proeyen, Phys. Rev. D 83, 025008 (2011).

[31] S. Ferrara, R. Kallosh, A. Linde, A. Marrani, and A. Van Proeyen, Phys. Rev. D 82, 045003 (2010).

[32] W. Buchmüller, V. Domcke, and K. Schmitz, J. Cosmol. Astropart. Phys. 04 (2013) 019.

[33] K. Ishiwata, Phys. Lett. B 782, 367 (2018).

[34] S. R. Coleman and E. J. Weinberg, Phys. Rev. D 7, 1888 (1973).

[35] T. Asaka, W. Buchmuller, and L. Covi, Phys. Lett. B 510, 271 (2001).

[36] A. Achucarro, J.-O. Gong, S. Hardeman, G. A. Palma, and S. P. Patil, J. Cosmol. Astropart. Phys. 01 (2011) 030.

[37] X. Chen and Y. Wang, J. Cosmol. Astropart. Phys. 04 (2010) 027.

[38] V. Domcke, K. Schmitz, and T. T. Yanagida, Nucl. Phys. B891, 230 (2015).

[39] V. Domcke and K. Schmitz, Phys. Rev. D 95, 075020 (2017).

[40] V. Domcke and K. Schmitz, Phys. Rev. D 97, 115025 (2018).

[41] F. Feruglio, arXiv:1706.08749.

[42] T. Kobayashi, K. Tanaka, and T. H. Tatsuishi, Phys. Rev. D 98, 016004 (2018).

[43] J. C. Criado and F. Feruglio, SciPost Phys. 5, 042 (2018).

[44] T. Kobayashi, N. Omoto, Y. Shimizu, K. Takagi, M. Tanimoto, and T. H. Tatsuishi, J. High Energy Phys. 11 (2018) 196.
[45] P. P. Novichkov, S. T. Petcov, and M. Tanimoto, Phys. Lett. B 793, 247 (2019).

[46] T. Nomura and H. Okada, Phys. Lett. B 797, 134799 (2019).

[47] T. Kobayashi, Y. Shimizu, K. Takagi, M. Tanimoto, and T. H. Tatsuishi, J. High Energy Phys. 02 (2020) 097.

[48] G.-J. Ding, S. F. King, and X.-G. Liu, J. High Energy Phys. 09 (2019) 074.

[49] T. Kobayashi, Y. Shimizu, K. Takagi, M. Tanimoto, and T. H. Tatsuishi, Phys. Rev. D 100, 115045 (2019); 101, 039904(E) (2020).

[50] T. Kobayashi, T. Nomura, and T. Shimomura, Phys. Rev. D 102, 035019 (2020).

[51] J. T. Penedo and S. T. Petcov, Nucl. Phys. B939, 292 (2019).

[52] P. P. Novichkov, J. T. Penedo, S. T. Petcov, and A. V. Titov, J. High Energy Phys. 04 (2019) 005.

[53] S. F. King and Y.-L. Zhou, Phys. Rev. D 101, 015001 (2020).

[54] P. P. Novichkov, J. T. Penedo, S. T. Petcov, and A. V. Titov, J. High Energy Phys. 04 (2019) 174.

[55] G.-J. Ding, S. F. King, and X.-G. Liu, Phys. Rev. D 100, 115005 (2019).

[56] T. Asaka, Y. Heo, T. H. Tatsuishi, and T. Yoshida, J. High Energy Phys. 01 (2020) 144.

[57] M.-C. Chen, S. Ramos-Sánchez, and M. Ratz, Phys. Lett. B 801, 135153 (2020).

[58] V. Kaplunovsky and J. Louis, Nucl. Phys. B444, 191 (1995).

[59] I. Antoniadis, E. Gava, K. S. Narain, and T. R. Taylor, Nucl. Phys. B432, 187 (1994).

[60] T. Asaka, Y. Heo, and T. Yoshida, Phys. Lett. B 811, 135956 (2020).

[61] Y. Gunji, K. Ishiwata, and T. Yoshida (to be published). 